\title{
Modeling the Evolution of Expectations and Uncertainty in General Equilibrium*
}

\author{
Francesco Bianchi \\ Cornell University \\ Duke University \\ CPER and NBER
}

\author{
Leonardo Melosi \\ Federal Reserve Bank of Chicago
}

This draft: February 2015

First draft: January 2012

\begin{abstract}
We develop methods to solve general equilibrium models in which forward-looking agents are subject to waves of pessimism, optimism, and uncertainty that turn out to critically affect macroeconomic outcomes. Agents in the model are fully rational, conduct Bayesian learning, and they know that they do not know. Therefore, agents take into account that their beliefs will evolve according to what they will observe. This framework accommodates both gradual and abrupt changes in beliefs and allows for an analytical characterization of uncertainty. We use a prototypical Real Business Cycle model to illustrate the methods.
\end{abstract}

JEL Codes: D83, C11, E22.

Keywords: Markov switching, general equilibrium models, uncertainty, Bayesian learning, rational expectations.

${ }^{*}$ We thank the editor Jesus Fernandez-Villaverde and two anonymous referees for excellent comments and suggestions. We also thank Fernando Alvarez, Gadi Barlevy, Jeff Campbell, Martin Eichenbaum, Eric Leeper, Alessandro Pavan, Monika Piazzesi, Martin Schneider, Dan Waggoner, and Tao Zha for very useful comments and discussions. We also wish to thank participants at Macro seminar of the Federal Reserve Bank of Chicago, Purdue University, the SED 2013 in South Korea, the Second Conference on Rational Inattention and Related Theories held at Oxford University. Todd Messer provided excellent research assistance. Francesco Bianchi gratefully acknowledges financial support from the National Science Foundation through grant SES-1227397. The views in this paper are solely the responsibility of the authors and should not be interpreted as reflecting the views of the Federal Reserve Bank of Chicago or any other person associated with the Federal Reserve System. Francesco Bianchi, Department of Economics, Duke University, 213 Social Sciences Building, Durham, NC, 27708-0089, USA. Leonardo Melosi, Federal Reserve Bank of Chicago, 230 South La Salle street, Chicago, IL 60604-1413, USA. Email: lmelosi@frbchi.edu. 


\section{Introduction}

A centerpiece of the rational expectations revolution is that economic outcomes critically depend on agents' beliefs about future events. Most general equilibrium models are solved assuming that agents have perfect knowledge about the stochastic properties of all the realized events. These are certainly strong restrictions imposed upon the dynamics of beliefs. For instance, the private sector is likely to have limited information about the future path of policymakers' decisions, the dynamics of dividend payments, or the likely duration of observed changes in the returns to labor and capital. These assumptions, in turn, influence the expectations formation mechanism and hence the predictions we draw from rational expectations models.

In this paper we develop methods to solve dynamic general equilibrium models in which forwardlooking and fully rational agents learn about the stochastic properties of realized events. This modeling framework captures waves of pessimism, optimism, and uncertainty that turn out to critically affect macroeconomic outcomes. Such outbursts of pessimism, optimism, and uncertainty may happen abruptly or may gradually unfold over a long period of time in response to the behavior of other agents or to the realizations of economic outcomes. Furthermore, this framework is well-suited to study the effects of shocks to beliefs and agents' uncertainty in Dynamic Stochastic General Equilibrium (DSGE) models. All results are derived within a modeling framework suitable for structural estimation that will allow researchers to bring the models to the data.

The evolution of agents' beliefs is modelled assuming the existence of different states of the world or regimes that differ according to the statistical properties of the exogenous shocks or based on the behavior of some of the agents in the model. Such regimes follow a Markov-switching process, which may be correlated with other aspects of the model. For example, the government could be more likely to inflate debt away when the level of spending is high. Agents are assumed to observe economic outcomes, but not the regimes themselves. Agents will then adopt Bayesian learning to infer which regime is in place. This will determine the evolution of agents' beliefs about future economic outcomes.

Our modeling framework does not rely on the assumption of anticipated utility that is often used in models characterized by a learning process. Such an assumption implies that agents forecast future events assuming that their beliefs will never change in the future. Instead, agents in our models know that they do not know. Therefore, when forming expectations, they take into account that their beliefs will evolve according to what they observe in the future. In our context, it is possible to go beyond the anticipated utility assumption because there are only a finite number of relevant beliefs and they are strictly linked to observable outcomes through the learning mechanism in a way that we can keep track of their evolution. It should also be noted that the proposed approach is based on agents being fully rational and hence their beliefs always being consistent in equilibrium. Rationality in our approach is essential in that it puts discipline on beliefs so as to make it possible to draw precise predictions from economic models.

The proposed model framework is flexible enough to encompass both abrupt and gradual changes in beliefs. For example, augmenting the modeling framework with signals about the regime in place allows one to capture the sharp effect of news on the evolution of the economy or to study the macroeconomic implications of changes in animal spirits about future events. At the same time, through the learning 
process, we can model situations in which agents' beliefs gradually change in response to the behavior of other agents or the realizations of stochastic events. This sluggish adjustment of public expectations is hard to reproduce through rational expectations models in which the functioning of the whole economy is common knowledge among agents. Furthermore, the methods introduced in this paper can be combined with techniques developed by Bianchi (2013a) to obtain an analytical characterization of the evolution of uncertainty. Bianchi (2013a) shows how to compute measures of expectations and uncertainty in Markovswitching models with perfect information. The combination of these methods endows researchers with a convenient toolkit to take dynamic general equilibrium rational expectations models to the growing amount of data on macroeconomic uncertainty.

We show how to apply these methods using a prototypical Real Business Cycle (RBC) model. In the model, total factor productivity (TFP) growth can assume two values: high or low. For each value of TFP growth, we allow for a long-lasting and a short-lasting regime. Therefore, while agents can observe the current TFP growth rate, they are uncertain about its future values, because they do not know if the current value is likely to last for a short time or for a long time. We consider a wide range of specifications, allowing for smooth transitions or abrupt changes in agents' optimism about future realizations of TFP growth. Each of these different specifications can be easily captured with the appropriate transition matrix governing the evolution of TFP growth. This has the important implication that the dynamics of pessimism, optimism, and uncertainty are consistent in equilibrium. Whenever a short-lasting regime is in fact realized, with the benefit of hindsight, agents' beliefs turn out to overreact to the regime change because agents always take into account the possibility that the economy entered a long-lasting regime. However, if, in fact, the regime is long-lasting, it takes time for agents' beliefs to line up with the actual realization. This implies that although agents are fully rational, their beliefs are generally misaligned with respect to the actual state of the economy. Such a misalignment is found to substantially influence consumption and capital allocation in the RBC model.

We show that the assumption of anticipated utility is not innocuous in the class of models studied in this paper. This is because anticipated utility leads to periods of overpessimism and overoptimism with respect to the case in which agents are fully rational. These mistakes cumulate over time because of agents' investment decisions with respect to physical capital accumulation. These findings are different from Cogley and Sargent (2008) who argue that there are only minor drawbacks stemming from the anticipated utility assumption as long as precautionary motives are not strong. Two reasons explain this discordance of results. First, the learning problem is different in the two papers. In Cogley and Sargent (2008) agents have to learn the transition matrix governing the evolution of regime changes, while in our case agents have to learn the regime that is in place. Second, they consider a model with no capital accumulation. In our setting, sluggish changes in physical capital imply that mistakes that were done in the past due to overpessimism or overoptimism accumulate over time and cannot be immediately undone.

The methods developed in this paper are based on the idea of expanding the number of regimes to take into account the learning mechanism. The central insight consists of recognizing that the evolution of agents' beliefs can be captured by defining an expanded set of regimes indexed with respect to agents' beliefs themselves. Once this structure has been imposed, the model can be recast as a Markov-switching dynamic stochastic general equilibrium (MS-DSGE) model with perfect information. If regime changes 
enter additively the model can be solved with standard solution methods such as gensys (Sims 2002) and Blanchard and Kahn (1980), following the approach described in Schorfheide (2005) and Liu et al. (2011). If instead regime changes enter multiplicatively the model can be solved with any of the methods developed for solving MS-DSGE models, such as Davig and Leeper (2007), Farmer et al. (2009), Cho (2014), and Foerster et al. (2013).

In both cases, the resulting solution is suitable for likelihood-based estimation. This is because even if the final number of regimes is very large, there is a tight link between observable outcomes and the evolution of agents' beliefs. In other words, the transition matrix governing the joint dynamics of the economy and agents' beliefs is highly restricted. For example, Bianchi and Melosi (2015) apply these methods and Bayesian techniques to estimate a model in which agents are uncertain about the future stance of monetary policy. This paper is therefore related to a growing literature that models parameter instability to capture changes in the evolution of the macroeconomy. This consists of two branches: Schorfheide (2005), Justiniano and Primiceri (2008), Bianchi (2013b), Davig and Doh (2014), and Fernandez-Villaverde and Rubio-Ramirez (2008) introduce parameter instability in DSGE models, while Sims and Zha (2006), Primiceri (2005), and Cogley and Sargent (2005) work with structural VARs. Finally, to the extent that we can model situations in which agents' beliefs evolve in response to policymakers' behavior, our work is also linked to papers that study how inflation expectations respond to policy decisions, such as Mankiw et al. (2004), Nimark (2008), Del Negro and Eusepi (2011), and Melosi (2014a b).

Schorfheide (2005) pioneers a method to estimate general equilibrium models in which agents learn the realization of a discrete Markov-switching process that affects the constants of the model-implied laws of motion. Specifically, they have to learn if the current central bank target for inflation is high or low. Our work sharply differs from this contribution. First, our framework can accommodate situations in which agents learn about regime changes that do not only affect the constant terms of the model, but also its autoregressive component. For example, Bianchi and Melosi (2015) use the proposed framework to estimate a model in which agents have to learn about future policymakers' behavior. Second, in our framework agents always have enough information to infer what the current state of the economy is or what other agents are doing: High or low growth, Hawkish or Dovish monetary policy, etc. Nevertheless, agents face uncertainty about the statistical properties of what they are observing. For example, agents could be uncertain about the persistence and the destination of a particular state. As we shall show, in a model in which agents are forward looking these sources of uncertainty have pervasive effects on the law of motion of the economy. Third, as previously pointed out, in our framework agents know that they do not know. In other words, when forming their expectations, they take into account that their beliefs will evolve according to what they will observe in the future. In Schorfheide (2005) agents take into account that the target will change over time, but they do not internalize how beliefs will evolve in response to that.

The application used in this paper is similar to the one considered by Edge et al. (2007), who study a dynamic general equilibrium model in which agents have to learn the persistence of realized shocks to the long-run growth rate. Our paper is different from that one in several respects. First, we develop a method that can be applied to all models in which agents have to learn the statistical properties of 
regime-switching parameters that enter the model additively (e.g., a constant as in our application and in the model studied by Edge et al. (2007)) as well as multiplicatively (e.g., the inflation parameter of a Taylor rule as in Bianchi and Melosi (2015)). Second, unlike our paper, Edge et al. (2007) do not discuss the implications for the dynamics of uncertainty.

Our approach mitigates a critical tension that often arises in the learning literature (e.g., Andolfatto and Gomme 2003) when agents are assumed to understand the structure of the economy. On the one hand, it would be desirable to have regimes that are very different in order to induce significant changes on economic dynamics. On the other hand, this would make the learning process relatively fast. Instead, in our approach even small differences in persistence have very large effects on agents' expectations because regimes may be still very different in terms of the probability of moving to alternative regimes. Therefore, our framework allows for both smooth or abrupt changes in agents' beliefs and for the possibility of signals that play the role of shocks to beliefs, affecting agents' expectations and uncertainty. Furthermore, our framework is suitable for likelihood estimation.

The remainder of the paper is organized as follows. Section 2 introduces the class of models and derives the main results. Section 3 applies the methods to an RBC model. In Section 4 , we discuss the main advantages of the proposed methodology. Section 5 concludes.

\section{The Model Framework}

In this section, we introduce the modeling environment to which our methods are applicable. The class of models we focus on has three salient features:

1. A model that can be expressed or approximated in the following form 1

$$
\Gamma_{0}\left(\xi_{t}\right) S_{t}=\Gamma_{c}\left(\xi_{t}\right)+\Gamma_{1}\left(\xi_{t}\right) S_{t-1}+\Psi\left(\xi_{t}\right) \varepsilon_{t}+\Pi \eta_{t}
$$

where $S_{t}$ is a vector containing all variables of the model known at time $t$ (including conditional expectations formed at time $t), \eta_{t}$ is a vector containing the endogenous expectation errors, and the random vector $\varepsilon_{t}$ contains the familiar Gaussian shocks. The hidden variable $\xi_{t}$ controls the parameter values in place at time, $\theta\left(\xi_{t}\right) \equiv\left\{\Gamma_{0}\left(\xi_{t}\right), \Gamma_{c}\left(\xi_{t}\right), \Gamma_{1}\left(\xi_{t}\right), \Psi\left(\xi_{t}\right)\right\}$, assumes discrete values $\xi_{t} \in\{1, \ldots, n\}$, and evolves according to a Markov-switching process with transition matrix $\mathcal{P}$.

2. Agents have to forecast the dynamics of the endogenous variables $S_{t+1}$ on the basis of Model (1) and their information set at time $t, \mathcal{I}_{t}$. This includes the history of model variables and shocks, but not the history of regimes, $\xi^{t}: \mathcal{I}_{t} \equiv\left\{S^{t}, \varepsilon^{t}\right\}$.

3. Some regimes are assumed to bring about the same model parameters, $\theta\left(\xi_{t}\right)$. Let us group the regimes into $m$ blocks $b_{j}=\left\{\xi_{t} \in\{1, \ldots, n\}: \theta\left(\xi_{t}\right)=\theta_{b_{j}}\right\}$, for $j \in\{1, \ldots, m\}$.

\footnotetext{
${ }^{1}$ The assumption of model linearity is not essential and can be actually relaxed as discussed in Subsection 4.3 . We focus on linear models because linearization is still the most popular approach to solve and estimate Markov-switching DSGE models.
} 
Given that agents know the structure of the model ( $\left.\begin{array}{lll}s u & 1\end{array}\right)$ and can observe the endogenous variables and the shocks ( sub 2), they can determine which set of parameter $\theta\left(\xi_{t}\right)$ is in place at each point in time. However, while this is enough for agents to establish the history of blocks, agents cannot exactly infer the realized regime $\xi_{t}$, because the regimes within each block share the same parameter values (sub 3), which makes the regimes within a block observationally equivalent to agents. Therefore the only distinguishable characteristics of a regime within a block are its stochastic properties, such as its relative persistence and the relative probability of switching to the regimes of the other blocks, which is captured by the transition matrix $P$. These characteristics are known to agents that will use them to learn about the regime in place today and to form expectations about the future. Therefore, points 1-3 describe a model in which agents learn about the latent variable $\xi_{t}$. As will be shown below, such a learning process affects the equilibrium law of motion of the economy. However, agents cannot extract any additional information about the underlying regime from observing the history of the endogenous variables $S^{t}$ because this reflects their own beliefs.

Henceforth, we will consider a benchmark case in which there are two blocks $(m=2)$ and two regimes within each block. This choice is made in order to keep notation simple. The extension to the case in which $m>2$ is straightforward. The probabilities of moving across regimes are summarized by the transition matrix:

$$
\mathcal{P}=\left[\begin{array}{ll|ll}
p_{11} & p_{12} & p_{13} & p_{14} \\
p_{21} & p_{22} & p_{23} & p_{24} \\
\hline p_{31} & p_{32} & p_{33} & p_{34} \\
p_{41} & p_{42} & p_{43} & p_{44}
\end{array}\right]
$$

in which the probability of switching to regime $j$ given that we are in regime $i$ is denoted by $p_{i j}$. Without loss of generality, we assume that regimes $\xi_{t}=1$ and $\xi_{t}=2$ belong to Block 1 , while regimes $\xi_{t}=3$ and $\xi_{t}=4$ belong to Block 2 . We consider only non-trivial blocks that satisfy $p_{11}+p_{12}+p_{21}+p_{22} \neq 0$ and $p_{33}+p_{34}+p_{43}+p_{44} \neq 0$. The excluded cases are trivial as both blocks would last only one period. Furthermore, we require that the two regimes that belong to the same block differ either in their persistence or in the probability of moving from one another; that is, we require that either $p_{11} \neq p_{22}$ or $p_{12} \neq p_{21}$ and either $p_{33} \neq p_{44}$ or $p_{34} \neq p_{43}$. This condition makes the within-block Bayesian learning non-trivial because it ensures that the transition submatrices within the two blocks differ, implying different statistical properties of the regimes within a block. This provides the rationale for agents to care about learning which regime they are in within a block. Finally, we will impose that $p_{11}+p_{22}>0$ and $p_{33}+p_{44}>0$. This last assumption guarantees that within a block at least one of the two regimes can last more than one period. Summarizing, for each block, we will maintain the following benchmark assumptions throughout the paper:

A1 Non-triviality assumption: $p_{11}+p_{12}+p_{21}+p_{22} \neq 0$ and $p_{33}+p_{34}+p_{43}+p_{44} \neq 0$.

A2 Non-trivial-learning assumption: Either $p_{11} \neq p_{22}$ or $p_{12} \neq p_{21}$ and either $p_{33} \neq p_{44}$ or $p_{34} \neq p_{43}$.

A3 Non-jumping assumption: $p_{11}+p_{22}>0$ and $p_{33}+p_{44}>0$. 
We will now proceed in two steps. First, in Subsection 2.1 we will characterize the evolution of agents' beliefs within a block for given prior beliefs. Second, in Subsection 2.2 we will explain how agents' beliefs are pinned down once the economy moves across blocks. The statistical properties of the economy, as captured by the transition matrix, will allow us to distinguish two cases: Static and Dynamic priors. It is worth emphasizing that in both cases the assumption of rationality will be maintained and all results will be based on the Bayes' theorem. Finally, for each of these cases, we will describe how to recast the model with information frictions as a perfect information rational expectations model obtained by expanding the number of regimes to keep track of agents' beliefs.

\subsection{Evolution of Beliefs Within a Block}

In what follows, we will derive the law of motion of agents' beliefs conditional on being in a specific block. The formulas derived below will provide a recursive law of motion for agents' beliefs based on Bayes' theorem. Such recursion applies for any starting values for agents' beliefs. These will be determined by agents' beliefs at the moment the system enters the new block. We will characterize these initial beliefs in the next subsection.

As we have noticed in the previous section, agents can infer the history of the blocks. Therefore, at each point in time, agents know the number of consecutive periods spent in the current block since the last switch. Let us denote the number of consecutive realizations of Block $i$ at time $t$ as $\tau_{t}^{i}, i \in\{1,2\}$. To fix ideas, suppose that the system is in Block 1 at time $t$, implying that $\tau_{t}^{1}>0$ and $\tau_{t}^{2}=0$. Then, there are only two possible outcomes for the next period. The economy can spend an additional period in Block 1, implying that $\tau_{t+1}^{1}=\tau_{t}^{1}+1$ and $\tau_{t+1}^{2}=0$, or it can move to Block 2, implying $\tau_{t+1}^{1}=0$ and $\tau_{t+1}^{2}=1$. In this subsection, we restrict our attention to the first case.

Using Bayes' theorem and the fact that $\operatorname{prob}\left(\xi_{t-1}=2 \mid \tau_{t-1}^{1}\right)=1-\operatorname{prob}\left(\xi_{t-1}=1 \mid \tau_{t-1}^{1}\right)$, the probability of being in Regime 1 given that we have observed $\tau_{t}^{1}$ consecutive realizations of Block $1, \operatorname{prob}\left(\xi_{t}=1 \mid \tau_{t}^{1}\right)$, is given by 2

$$
\operatorname{prob}\left(\xi_{t}=1 \mid \tau_{t}^{1}\right)=\frac{\operatorname{prob}\left(\xi_{t-1}=1 \mid \tau_{t-1}^{1}\right)\left(p_{11}-p_{21}\right)+p_{21}}{\operatorname{prob}\left(\xi_{t-1}=1 \mid \tau_{t-1}^{1}\right)\left(p_{11}+p_{12}-p_{21}-p_{22}\right)+p_{21}+p_{22}}
$$

where $\tau_{t}^{1}=\tau_{t-1}^{1}+1$ and for $\tau_{t}^{1}>1$. Notice that for $\tau_{t}^{1}=1, \operatorname{prob}\left(\xi_{t}=1 \mid \tau_{t}^{1}\right)$ denotes the initial beliefs that will be discussed in Subsection 2.2. Equation (3) is a rational first-order difference equation that allows us to recursively characterize the evolution of agents' beliefs about being in Regime 1 while the system is in Block 1. The probability of being in Regime 3 given that we have observed $\tau_{t}^{2}$ consecutive realizations of Block $2, \operatorname{prob}\left(\xi_{t}=3 \mid \tau_{t}^{2}\right)$, can be analogously derived:

$$
\operatorname{prob}\left(\xi_{t}=3 \mid \tau_{t}^{2}\right)=\frac{\operatorname{prob}\left(\xi_{t-1}=3 \mid \tau_{t-1}^{2}\right)\left(p_{33}-p_{43}\right)+p_{43}}{\operatorname{prob}\left(\xi_{t-1}=3 \mid \tau_{t-1}^{2}\right)\left(p_{33}+p_{34}-p_{43}-p_{44}\right)+p_{43}+p_{44}} .
$$

where $\tau_{t}^{2}=\tau_{t-1}^{2}+1$ and for $\tau_{t}^{2}>1$.

The recursive equations (3) and (4) characterize the dynamics of agents' beliefs in both blocks for a

\footnotetext{
${ }^{2}$ A detailed derivation of equation $\sqrt{3}$ is provided in Appendix A
} 
given set of prior beliefs. The following proposition states that under the benchmark assumptions A1-A3, these recursive equations converge. This convergence result will be key to being able to recast Model (1)-(2) in terms of a finite dimensional set of regimes indexed with respect to agents' beliefs.

Proposition 1 Convergence. Under the benchmark assumptions A1-A3, for any $\varepsilon>0$ there exists a $\tau_{1}^{*} \in \mathbb{N}$ and $\tau_{2}^{*} \in \mathbb{N}$ such that:

$$
\begin{aligned}
& \operatorname{prob}\left(\xi_{t}=1 \mid \tau_{1}^{*}\right)-\operatorname{prob}\left(\xi_{t}=1 \mid \tau_{1}^{*}+1\right)<\varepsilon \\
& \operatorname{prob}\left(\xi_{t}=3 \mid \tau_{2}^{*}\right)-\operatorname{prob}\left(\xi_{t}=3 \mid \tau_{2}^{*}+1\right)<\varepsilon
\end{aligned}
$$

\section{Proof. See Appendix B.}

In what follows, we denote the converging probabilities for $\operatorname{prob}\left(\xi_{t}=1 \mid \tau_{1}\right)$ and $\operatorname{prob}\left(\xi_{t}=3 \mid \tau_{2}\right)$ as $\tilde{\lambda}_{b_{1}}$ and $\tilde{\lambda}_{b_{2}}$, respectively.

\section{$2.2 \quad$ Evolution of Beliefs Across Blocks}

In the previous subsection, we characterized the evolution of agents' beliefs conditional on being in a specific block. The formulas derived above apply to any set of initial beliefs. In this subsection, we will pin down agents' beliefs at the moment the economy moves across blocks. These beliefs will serve as starting points for the recursions (3) and (4) governing the evolution of beliefs within a block.

Suppose for a moment that before switching to the new block, agents could observe the regime that was in place in the old block. Notice that in this case the transition matrix conveys all the information necessary to pin down agents' prior beliefs about the regime in place within the new block. Specifically, we have that if the economy moves from Block 2 to Block 1, the probability of being in Regime 1 is given by

$$
\operatorname{prob}\left(\xi_{t}=1 \mid \xi_{t-1}=3, \tau_{t}^{1}=1\right)=\frac{p_{31}}{p_{31}+p_{32}},
$$

if the economy was under Regime 3 in the previous period, or by

$$
\operatorname{prob}\left(\xi_{t}=1 \mid \xi_{t-1}=4, \tau_{t}^{1}=1\right)=\frac{p_{41}}{p_{41}+p_{42}}
$$

if the economy was under Regime 4 in the previous period. Symmetrically, the probability of being in Regime 3 given that the economy just moved to Block 2 is given by

$$
\operatorname{prob}\left(\xi_{t}=3 \mid \xi_{t-1}=1, \tau_{t}^{2}=1\right)=\frac{p_{13}}{p_{13}+p_{14}}
$$

if the economy was under Regime 1 in the previous period, or by

$$
\operatorname{prob}\left(\xi_{t}=3 \mid \xi_{t-1}=2, \tau_{t}^{2}=1\right)=\frac{p_{23}}{p_{23}+p_{24}}
$$

if the economy was previously under Regime 2 .

However, in the model, agents never observe the regime that is in place. Therefore, their beliefs at the moment the economy moves from one block to the other will be a weighted average of the probabilities 
outlined above. The weights, in turn, will depend on agents' beliefs at the moment of the switch. In what follows we will focus on three cases:

1. Static prior beliefs. In this case, the transition matrix $\mathcal{P}$ is such that every time the economy enters a new block, agents' beliefs about which regime has been realized do not depend on their beliefs right before the switch. Thus, what has been observed in the past block does not help rational agents to form expectations in the new block. Such a transition matrix has the virtue of delivering a nice closed-form analytical characterization for the dynamics of beliefs $3^{3}$

2. Dynamic prior beliefs. In this case, the transition matrix $\mathcal{P}$ is such that beliefs about which regime is prevailing within a block affect prior beliefs the moment the economy moves to the new block.

3. Signals. Exogenous signals $\varpi_{t}$ about the current regime are also observed by agents. Signals are assumed to be distributed according to $p\left(\varpi_{t} \mid \xi_{t}\right)$.

It is worth clarifying that nothing prevents the researcher from combining the three cases described above. For example, static prior beliefs could characterize one block but not another or agents could receive a signal every time the economy enters a new block.

\subsubsection{The Case of Static Prior Beliefs}

In the case of static prior beliefs, the transition matrix $\mathcal{P}$ implies that every time the system enters a new block, rational agents' beliefs are the same regardless of the history of past beliefs. It is immediate to show that necessary and sufficient conditions for this to happen are:

$$
\begin{aligned}
& \operatorname{prob}\left(\xi_{t}=1 \mid \xi_{t-1}=3, \tau_{t}^{1}=1\right)=\frac{p_{31}}{p_{31}+p_{32}}=\frac{p_{41}}{p_{41}+p_{42}}=\operatorname{prob}\left(\xi_{t}=1 \mid \xi_{t-1}=4, \tau_{t}^{1}=1\right) \\
& \operatorname{prob}\left(\xi_{t}=3 \mid \xi_{t-1}=1, \tau_{t}^{2}=1\right)=\frac{p_{13}}{p_{13}+p_{14}}=\frac{p_{23}}{p_{23}+p_{24}}=\operatorname{prob}\left(\xi_{t}=3 \mid \xi_{t-1}=2, \tau_{t}^{2}=1\right)
\end{aligned}
$$

In other words, the transition matrix $\mathcal{P}$ is such that when the economy leaves a block, the relative probability of the two regimes in the new block is not affected by the regime that was in place before. Agents' beliefs are uniquely pinned down by (5) and (6) because agents are fully rational and know the transition matrix governing the evolution of regimes.

The recursive equations (3) and (4) combined with the initial conditions (5) and (6) uniquely characterize the dynamics of agents' beliefs in each block. To see this, notice that for each block, there is a unique path for the evolution of agents' beliefs, given that (5) and (6) make agents' beliefs before entering the block irrelevant. Furthermore, Proposition 1 guarantees that there exists a $\tau_{1}^{*} \in \mathbb{N}$ and $\tau_{2}^{*} \in \mathbb{N}$ such that agents' beliefs converge for an arbitrary level of accuracy. Therefore, in the case of static priors the number of consecutive periods spent in a block $\left(\tau_{t}^{i}\right)$ is a sufficient statistic to pin down the dynamics of beliefs in both blocks. Equipped with this important result, we can re-cast Model (1)-(2) in terms of a new set of regimes indexed with respect to the number of consecutive periods spent in a block $\tau_{t}^{i}$, $i \in\{1,2\}$ :

\footnotetext{
${ }^{3}$ A simplified version of this problem with three regimes is studied by Barlevy (1998) within a partial equilibrium framework.
} 


$$
\Gamma_{0}\left(\tau_{t}\right) S_{t}=\Gamma_{c}\left(\tau_{t}\right)+\Gamma_{1}\left(\tau_{t}\right) S_{t-1}+\Psi\left(\tau_{t}\right) \varepsilon_{t}+\Pi \eta_{t}
$$

where $\varepsilon_{t} \sim N\left(0, \Sigma_{\varepsilon}\right)$ is a vector of exogenous Gaussian shocks, $\eta_{t}$ is a vector of endogenous expectation errors, and the $\tau_{1}^{*}+\tau_{2}^{*}$ regimes $\tau_{t} \equiv\left(\tau_{t}^{1}, \tau_{t}^{2}\right)$ evolve according to the transition matrix

$$
\widetilde{\mathcal{P}}=\left[\begin{array}{ll}
\widetilde{\mathcal{P}}_{11} & \widetilde{\mathcal{P}}_{12} \\
\widetilde{\mathcal{P}}_{21} & \widetilde{\mathcal{P}}_{22}
\end{array}\right],
$$

where the matrices $\widetilde{\mathcal{P}}_{11}$ and $\widetilde{\mathcal{P}}_{12}$ are given by

$$
\begin{gathered}
\widetilde{\mathcal{P}}_{11} \equiv\left[\begin{array}{cccccc}
0 & \operatorname{prob}\left\{\tau_{t+1}^{1}=2 \mid \tau_{t}^{1}=1\right\} & 0 & \ldots & 0 & 0 \\
0 & 0 & \operatorname{prob}\left\{\tau_{t+1}^{1}=3 \mid \tau_{t}^{1}=2\right\} & \ldots & 0 & 0 \\
\vdots & \vdots & \vdots & \ddots & \vdots & \vdots \\
0 & 0 & 0 & \ldots & 0 & \operatorname{prob}\left\{\tau_{t}^{1}=\tau^{*} \mid \tau_{t}^{1}=\tau^{*}-1\right\} \\
0 & 0 & 0 & \ldots & 0 & \operatorname{prob}\left\{\tau_{t+1}^{1}>\tau^{*} \mid \tau_{t}^{1}=\tau^{*}\right\}
\end{array}\right] \\
\widetilde{\mathcal{P}}_{12} \equiv\left[\begin{array}{cc}
1-\operatorname{prob}\left\{\tau_{t+1}^{1}=2 \mid \tau_{t}^{1}=1\right\} & \mathbf{0}_{1 \times\left(\tau^{*}-1\right)} \\
\vdots & \vdots \\
1-\operatorname{prob}\left\{\tau_{t+1}^{1}>\tau^{*} \mid \tau_{t}^{1}=\tau^{*}\right\} & \mathbf{0}_{1 \times\left(\tau^{*}-1\right)}
\end{array}\right]
\end{gathered}
$$

with the elements of the matrices given by

$$
\operatorname{prob}\left\{\tau_{t+1}^{i}=\tau_{t}^{i}+1 \mid \tau_{t}^{i}\right\}=\operatorname{prob}\left(\xi_{t}=1 \mid \tau_{t}^{1}\right)\left(p_{11}+p_{12}\right)+\left(1-\operatorname{prob}\left(\xi_{t}=1 \mid \tau_{t}^{1}\right)\right)\left(p_{21}+p_{22}\right)
$$

where $\operatorname{prob}\left(\xi_{t}=1 \mid \tau_{t}^{1}\right)$ can be obtained from the recursive equation (3) and equation (5). The matrices $\widetilde{\mathcal{P}}_{21}$ and $\widetilde{\mathcal{P}}_{22}$ can be analogously derived.

Let us make a simple example to clarify the form of the transition matrix $\widetilde{\mathcal{P}}$. Assume that the system switches from Block 2 to Block 1 at time $t$; that is, the number of consecutive periods in Block 1 is one $\left(\tau_{t}^{1}=1\right)$, which corresponds to the first regime of Block 1 . The first row of the transition matrix $\widetilde{\mathcal{P}}$ tells us that there are only two possible events that can occur in period $t+1$. First, the system stays in Block 1 and hence it moves to the second regime of Block $1\left(\tau_{t+1}^{1}=2\right)$. This is captured by the nonzero probability $\operatorname{prob}\left\{\tau_{t+1}^{1}=2 \mid \tau_{t}^{1}=1\right\}$, which is defined in equation (8) and positioned on the second column of the submatrix $\widetilde{\mathcal{P}}_{11}$. The second possible event is that the system switches to Block 2 and hence moves to the first regime of that block $\left(\tau_{t+1}^{2}=1\right)$. This event is captured by the non-zero probability $\left(1-\operatorname{prob}\left\{\tau_{t+1}^{1}=2 \mid \tau_{t}^{1}=1\right\}\right)$ positioned on the first column of the submatrix $\widetilde{\mathcal{P}}_{12}$. Since there are the only two possible events that can be realized in period $t+1$, all other elements of the first row of the matrix $\widetilde{\mathcal{P}}$ are zero. As the system keeps staying in Block 1, we transit to the next higher regime of the block until we reach a number of period in the block that is equal to or larger than the truncation point $\tau^{*}$. This within-block evolution of regimes is captured by the form of the diagonal submatrices $\widetilde{\mathcal{P}}_{11}$ and $\widetilde{\mathcal{P}}_{22}$ whose non-zero probabilities are off-diagonal, except for the one located on the $\tau^{*}-t h$ row. As the system switches to Block 2, we move to the first regime of Block 2 regardless of the regime of Block 1 the 
system comes from. This is due to the static feature of the prior beliefs in this example and is captured

by the form of the off-diagonal submatrices $\widetilde{\mathcal{P}}_{12}$ and $\widetilde{\mathcal{P}}_{21}$, in which the non-zero probabilities are all on their first column.

Notice that the newly defined set of regimes keeps track of both the parameters in place at each point in time and the evolution of agents' beliefs. Since Model (7) is a Markov-switching DSGE model with perfect information, it can be solved using the techniques developed by Schorfheide (2005), Liu et al. (2011), Davig and Leeper (2007), Farmer et al. (2009), Cho (2014), and Foerster et al. (2013). The result is an MS-VAR in the DSGE state vector $S_{t}$ :

$$
S_{t}=c\left(\tau_{t}, \widetilde{\mathcal{P}}\right)+T\left(\tau_{t}, \widetilde{\mathcal{P}}\right) S_{t-1}+R\left(\tau_{t}, \widetilde{\mathcal{P}}\right) \varepsilon_{t}
$$

where the law of motion of the economy depends on agents' beliefs as captured by $\tau_{t}$. With the results of Proposition 1 at hand, the solution of Model (7) with a truncated number of regimes $\tau_{t}$ approximates the solution of the original model (1) with learning. Notice that the accuracy of this approximation can be made arbitrarily precise simply by increasing the number of regimes $\tau^{*}$. Furthermore, it is worth pointing out that in the case of static priors the approximation error stems only from truncating agents' learning process. For all regimes such that $\tau_{t}^{i}<\tau_{i}^{*}$ agents' beliefs exactly coincide with the analytical values derived using (3) and (4) and conditions (5) and (6).

It is important to notice that the case of static prior belief does not constitute a deviation from rationality or from the Bayes theorem. In fact, it directly stems from the application of the Bayes theorem. Given condition (5), agents would be irrational to have different beliefs when the system enters Block 1 in different periods. Condition (6) leads to this exact implication all the times the system enters Block 2. We single out the case of static prior because when conditions (5)-(6) hold, the approximation error from truncating agents' learning process is negligible. This is for two reasons. First, Proposition 1 ensures that the change in agents' beliefs is negligible once the system has spent a sufficiently long period of time in a block. Second, the initial beliefs can be pinned down analytically thanks to conditions (5) $-(6)$.

\subsubsection{The Case of Dynamic Prior Beliefs}

When conditions (5) and (6) do not hold, past beliefs always influence current beliefs. In this case, the number of consecutive periods $\tau_{t}$ spent in a block is no longer a sufficient statistic for agents' beliefs. However, as pointed out before, the recursive equations (3) and (4) hold for any prior beliefs. Therefore, these equations still capture the dynamics of beliefs while the system stays in a block. Furthermore, it follows that the sufficient conditions for convergence derived in Subsection 2.1 still apply. Nevertheless, the initial conditions are now different from (5) and (6) as they will depend on beliefs in the past block. Specifically, agents' starting beliefs upon the shift from Block 2 to Block 1 are given by

$$
\operatorname{prob}\left\{\xi_{t}=1 \mid \mathcal{I}_{t}\right\}=\frac{\operatorname{prob}\left\{\xi_{t-1}=3 \mid \mathcal{I}_{t-1}\right\} p_{31}+\left(1-\operatorname{prob}\left\{\xi_{t-1}=3 \mid \mathcal{I}_{t-1}\right\}\right) p_{41}}{\operatorname{prob}\left\{\xi_{t-1}=3 \mid \mathcal{I}_{t-1}\right\}\left(p_{31}+p_{32}\right)+\left(1-\operatorname{prob}\left\{\xi_{t-1}=3 \mid \mathcal{I}_{t-1}\right\}\right)\left(p_{41}+p_{42}\right)}
$$


while if the system just entered Block 2, starting beliefs read

$$
\operatorname{prob}\left\{\xi_{t}=3 \mid \mathcal{I}_{t}\right\}=\frac{\operatorname{prob}\left\{\xi_{t-1}=1 \mid \mathcal{I}_{t-1}\right\} p_{13}+\left(1-\operatorname{prob}\left\{\xi_{t-1}=1 \mid \mathcal{I}_{t-1}\right\}\right) p_{23}}{\operatorname{prob}\left\{\xi_{t-1}=1 \mid \mathcal{I}_{t-1}\right\}\left(p_{13}+p_{14}\right)+\left(1-\operatorname{prob}\left\{\xi_{t-1}=1 \mid \mathcal{I}_{t-1}\right\}\right)\left(p_{23}+p_{24}\right)}
$$

Notice that, using their information set $\mathcal{I}_{t}$, agents can keep track of both the number of consecutive deviations and their starting beliefs. Therefore, in the case of dynamic prior beliefs two variables pin down the dynamics of beliefs over time: how many consecutive periods the system has spent in the current block and the initial beliefs agents had when the system entered the current block.

Note that in the case of static priors, beliefs get reinitialized as the system switches to a new block. In fact, as a switch to a new block occurs, agents' beliefs are always the same and given by equations (5) and (6). In the case of dynamic priors, these initial probabilities depend on the entire history and hence they change over time. This feature makes it impossible to analytically pin down the evolution of beliefs across blocks: There are infinite possible paths and as a result infinite possible values for agents' beliefs.

Let us assume that we can overcome this infinite dimensionality problem by approximating the dynamics of beliefs using a finite number of regimes $\zeta_{t}$ and the associated transition matrix $\widehat{\mathcal{P}}$ so that the original Model (1) can be recast in terms of the new set of regimes $\zeta_{t}$ :

$$
\Gamma_{0}\left(\zeta_{t}\right) S_{t}=\Gamma_{c}\left(\zeta_{t}\right)+\Gamma_{1}\left(\zeta_{t}\right) S_{t-1}+\Psi\left(\zeta_{t}\right) \varepsilon_{t}+\Pi \eta_{t}
$$

Therefore, the task of solving the model with learning in (1)-(2) boils down to solving the perfectinformation model (12) using available solution algorithms for MS-DSGE models. The resulting law of motion is once again an MS-VAR:

$$
S_{t}=c\left(\zeta_{t}, \widehat{\mathcal{P}}\right)+T\left(\zeta_{t}, \widehat{\mathcal{P}}\right) S_{t-1}+R\left(\zeta_{t}, \widehat{\mathcal{P}}\right) \varepsilon_{t}
$$

\subsubsection{A Grid-Based Approach}

We tackle the issue of tracking beliefs when agents' priors are dynamic by defining a set of regimes based on a grid that approximates agents' beliefs at each point in time. Denote the grid for beliefs prob $\left\{\xi_{t}=1 \mid \mathcal{I}_{t}\right\}$ as $\mathcal{G}_{b_{1}}=\left\{\mathcal{G}_{1}, \ldots, \mathcal{G}_{g_{1}}\right\}$ and for beliefs $\operatorname{prob}\left\{\xi_{t}=3 \mid \mathcal{I}_{t}\right\}$ as $\mathcal{G}_{b_{2}}=\left\{\mathcal{G}_{g_{1}+1}, \ldots, \mathcal{G}_{g_{1}+g_{2}}\right\}$ where $0 \leq \mathcal{G}_{i} \leq 1$, all $1 \leq i \leq g=g_{1}+g_{2}$. Furthermore, we denote the whole grid as $\mathcal{G}=\mathcal{G}_{b 1} \cup \mathcal{G}_{b 2}$. Endowed with such a grid, we can recast the original model in terms of a new set of regimes $\zeta_{t} \in\left\{1, \ldots, g_{1}+g_{2}\right\}$, any $t$. The new regime $\zeta_{t}$ captures the knot of the grid $\mathcal{G}$ that best approximates agents' beliefs; that is, in our notation $\operatorname{prob}\left\{\xi_{t}=1 \mid \mathcal{I}_{t}\right\}$ when the system is in Block 1 and $\operatorname{prob}\left\{\xi_{t}=3 \mid \mathcal{I}_{t}\right\}$ when the system is in Block 2. The transition probability matrix for these new regimes can be pinned down using the recursions (3) and (4) and the initial conditions $(10)$ and (11). The algorithm below illustrates how exactly to perform this task.

Algorithm Initialize the transition matrix $\widehat{\mathcal{P}}$ for the new regimes $\zeta_{t}$, setting $\widehat{\mathcal{P}}=\mathbf{0}_{g \times g}$.

Step 1 For each of the two blocks, do the following steps (without loss of generality we describe the steps for Block 1): 
Step 1.1 For any grid point $\mathcal{G}_{i} \in \mathcal{G}_{b_{1}}, 1 \leq i \leq g_{1}$, compute

$$
\widehat{\mathcal{P}}(i, j)=\operatorname{prob}\left\{\xi_{t-1}=1 \mid \mathcal{I}_{t-1}\right\}\left(p_{11}+p_{12}\right)+\left(1-\operatorname{prob}\left\{\xi_{t-1}=1 \mid \mathcal{I}_{t-1}\right\}\right)\left(p_{21}+p_{22}\right)
$$

where $\operatorname{prob}\left\{\xi_{t-1}=1 \mid \mathcal{I}_{t-1}\right\}=\mathcal{G}_{i}$ and $j \leq g_{1}$ is set so as to min $\left|\operatorname{prob}\left\{\xi_{t}=1 \mid \mathcal{I}_{t}\right\}-\mathcal{G}_{j}\right|$, where $\operatorname{prob}\left\{\xi_{t}=1 \mid \mathcal{I}_{t}\right\}$ is computed using the recursive equation $(3)$ by approximating prob $\left\{\xi_{t-1}=1 \mid \mathcal{I}_{t-1}\right\}=$ $\mathcal{G}_{i}$. To ensure the convergence of beliefs, we correct $j$ as follows: if $j=i$ and $\mathcal{G}_{i} \neq \widetilde{\lambda}_{b_{1}}$, then set $j=\min \left(j+1, g_{1}\right)$ if $\mathcal{G}_{i}<\widetilde{\lambda}_{b_{1}}$ or $j=\max (1, j-1)$ if $\mathcal{G}_{i}>\widetilde{\lambda}_{b_{1}}$.

Step 1.2 For any grid point $\mathcal{G}_{i} \in \mathcal{G}_{b_{1}}, 1 \leq i \leq g_{1}$, compute $\widehat{\mathcal{P}}(i, l)=1-\widehat{\mathcal{P}}(i, j)$ with $l>g_{1}$ satisfying

$$
\min \left|\frac{\operatorname{prob}\left\{\xi_{t-1}=1 \mid \mathcal{I}_{t-1}\right\} p_{13}+\left(1-\operatorname{prob}\left\{\xi_{t-1}=1 \mid \mathcal{I}_{t-1}\right\}\right) p_{23}}{\operatorname{prob}\left\{\xi_{t-1}=1 \mid \mathcal{I}_{t-1}\right\}\left(p_{13}+p_{14}\right)+\left(1-\operatorname{prob}\left\{\xi_{t-1}=1 \mid \mathcal{I}_{t-1}\right\}\right)\left(p_{23}+p_{24}\right)}-\mathcal{G}_{l}\right|
$$

where $\operatorname{prob}\left\{\xi_{t-1}=1 \mid \mathcal{I}_{t-1}\right\}=\mathcal{G}_{i}$.

Step 2 If no column of $\widehat{\mathcal{P}}$ has all zero elements, stop. Otherwise, go to Step 3.

Step 3 Construct the matrix $T$ as follows. Set $j=1$ and $l=1$. While $j \leq g$, if $\sum_{i=1}^{g} \widehat{\mathcal{P}}(i, j)=0$ set $j=j+1$. Otherwise, if $\sum_{i=1}^{g} \widehat{\mathcal{P}}(i, j) \neq 0$ : (1) set $T(j, l)=1,(2)$ set $T(j, v)=0$ for any $1 \leq v \leq g$ and $v \neq l,(3)$ set $l=l+1$ and $j=j+1$.

Step 4 Write the transition equation as $\widehat{\mathcal{P}}^{R}=T \cdot \widehat{\mathcal{P}} \cdot T^{\prime}$. If no column of $\widehat{\mathcal{P}}^{R}$ has all zero elements, set $\widehat{\mathcal{P}}=\widehat{\mathcal{P}}^{R}$ and stop. Otherwise, go to step 3 .

Step 1.1 determines the regime $j$ the system will go to if it stays in Block 1 next period and fills up the appropriate element $(i, j)$ of the transition matrix $\widehat{\mathcal{P}}$ with the probability of moving to Regime $j$. Step 1.2 computes the regime $l$ the system will go to if it leaves Block 1 and fills up the appropriate element $(i, l)$ of matrix $\widehat{\mathcal{P}}$. Steps $2-4$ are not necessary but help to keep the dimension of the grid small, getting rid of regimes that will never be reached. For computational convenience, we always add the convergence points for the two blocks (i.e., $\widetilde{\lambda}_{b_{1}}$ in the case of Block 1) to the grid $\mathcal{G}$. On many occasions, it is a good idea to make the grid near the convergence knot very fine to improve the precision of the approximation.

The task of solving the model with learning in (1)-(2) boils down to solving the perfect-information model (12) using available solution algorithms for MS-DSGE models ${ }^{4}$

\subsubsection{Signals}

Let us assume that agents observe signals about the realized regime. To fix notation, denote the signal received at time $t$ as $\varpi_{t}$ and, for simplicity, assume that it can have only two values, 1 or 2 . We denote the history of signals received up to time $t$ as $\varpi^{t}=\left\{\varpi_{1}, \ldots, \varpi_{t}\right\}$. We denote the probability that the signal is equal to $q \in\{1,2\}$, conditional on the regime being equal to $h \in\{1,2,3,4\}$ as $\operatorname{prob}\left\{\varpi_{t}=q \mid \xi_{t}=h\right\}$.

\footnotetext{
${ }^{4}$ In Subsection 3.2.1 we present an example for which both the approximation error and the time required to solve the model turn out to be very small.
} 
The model with signals can be solved by introducing a new system of regimes $\zeta_{t}$, which indexes the grid points corresponding to the probabilities $\operatorname{prob}\left\{\xi_{t}=1 \mid \mathcal{I}_{t}, \varpi^{t}\right\}$ and $\operatorname{prob}\left\{\xi_{t}=3 \mid \mathcal{I}_{t}, \varpi^{t}\right\}$, and following the same logic used in the previous subsection. As we shall show, signals will allows us to introduce shocks to beliefs; that is, exogenously driven changes in beliefs that are not associated with changes in the economy's state $\xi_{t}$.

To fill up the transition matrix $\widehat{\mathcal{P}}$ for the new set of regimes, one can implement the algorithm detailed in Subsection 2.2.2 with only the little tweak of updating beliefs using the information contained in the observed signal. For instance, we compute the ex-post-probability $\operatorname{prob}\left(\xi_{t}=1 \mid \mathcal{I}_{t}, \varpi^{t}\right)$

$$
\operatorname{prob}\left(\xi_{t}=1 \mid \mathcal{I}_{t}, \varpi^{t-1}, \varpi_{t}=q\right)=\frac{\operatorname{prob}\left(\varpi_{t}=q \mid \xi_{t}=1\right) \operatorname{prob}\left(\xi_{t}=1 \mid \mathcal{I}_{t}, \varpi^{t-1}\right)}{\sum_{i=1}^{2} \operatorname{prob}\left(\varpi_{t}=q \mid \xi_{t}=i\right) \operatorname{prob}\left(\xi_{t}=i \mid \mathcal{I}_{t}, \varpi^{t-1}\right)}, q \in\{1,2\}
$$

where $\operatorname{prob}\left(\xi_{t}=1 \mid \mathcal{I}_{t}, \varpi^{t-1}\right)$ is computed using the recursive equation (3) for a given initial point in the $\operatorname{grid} \mathcal{G}$ that approximates $\operatorname{prob}\left(\xi_{t-1}=1 \mid \mathcal{I}_{t-1}, \varpi^{t-1}\right)$. We use the probability computed in equation (14) to determine the appropriate destination points of the grid $\mathcal{G}$, which we denote as $j_{q}, q \in\{1,2\}$. Note that for any given initial belief $\operatorname{prob}\left(\xi_{t-1}=1 \mid \mathcal{I}_{t-1}, \varpi^{t-1}\right) \in \mathcal{G}$, the (ex-post) belief $\operatorname{prob}\left(\xi_{t}=1 \mid \mathcal{I}_{t}, \varpi^{t-1}, \varpi_{t}=q\right)$ now pins down the grid points, depending on the realization of the signal $\varpi_{t}$. Once these two destination points in the grid are determined, we can fill up the transition probability as follows:

$$
\widehat{\mathcal{P}}\left(i, j_{q}\right)=\sum_{v=1}^{2} \operatorname{prob}\left\{\xi_{t}=v \mid \mathcal{I}_{t-1}, \varpi^{t-1}\right\} \operatorname{prob}\left\{\varpi_{t}=q \mid \xi_{t}=v\right\}, \quad q \in\{1,2\}
$$

where

$$
\operatorname{prob}\left\{\xi_{t}=v \mid \mathcal{I}_{t-1}, \varpi^{t-1}\right\}=\sum_{u=1}^{2} \operatorname{prob}\left\{\xi_{t-1}=u \mid \mathcal{I}_{t-1}, \varpi^{t-1}\right\} p_{u v}
$$

and we approximate $\operatorname{prob}\left\{\xi_{t-1}=1 \mid \mathcal{I}_{t-1}, \varpi^{t-1}\right\} \in \mathcal{G}$. Note that in the case of binary signals, each row of the transition matrix $\widehat{\mathcal{P}}$ has up to four non-zero elements. This completes the derivation of the submatrix $\widehat{\mathcal{P}}_{11}$, which governs the evolution of beliefs within Block 1 . How to obtain the other submatrices $\widehat{\mathcal{P}}_{12}$, $\widehat{\mathcal{P}}_{21}$, and $\widehat{\mathcal{P}}_{22}$ is detailed in Appendix $\mathrm{C}$.

\section{Applications}

In this section, we introduce a prototypical RBC model to illustrate the properties of the methods detailed above. Central to our discussion will be the evolution of optimism and pessimism and the implications thereof for consumption and saving decisions. The representative household chooses the sequence of consumption $c_{t}$ and capital $k_{t}$ :

$$
\max _{c_{t}, k_{t}} \widetilde{E}_{0} \sum_{t=0}^{\infty} \beta^{t} \ln c_{t}
$$

subject to the resource constraint $c_{t}+k_{t}=z_{t} k_{t-1}^{\alpha}+(1-\delta) k_{t-1}$ with $\alpha<1$ and $0<\delta<1$. Let $\widetilde{E}_{t}(\cdot)$ denote the expectation operator conditional on households' information set at time $t$. We assume that total factor productivity (TFP) $z_{t}$ follows an exogenous process, such that

$$
\ln z_{t}=\mu\left(\xi_{t}\right)+\ln z_{t-1}+\sigma_{z} \varepsilon_{t}
$$


where $\varepsilon_{t} \stackrel{\text { iid }}{\sim} N(0,1)$ and $\xi_{t}$ denotes a discrete Markov process affecting the drift of TFP. This process evolves according to the transition probability matrix $\mathcal{P}$. We assume that $\xi_{t}$ can take four values; that is, $\xi_{t} \in\{1,2,3,4\}$. These values map into values for the TFP drift $\mu\left(\xi_{t}\right)$ as follows $\xi_{t} \in\{1,2\} \Longrightarrow \mu_{t}\left(\xi_{t}\right)=$ $\mu_{H}$ and $\xi_{t} \in\{3,4\} \Longrightarrow \mu_{t}\left(\xi_{t}\right)=\mu_{L}$, where $\mu_{L}<\mu_{H}$. In Block 1, Regimes 1 and 2 differ in their likely persistence: $p_{11}<p_{22}$. The same applies to Regimes 3 and 4 in Block 2: $p_{33}<p_{44}$. We call Regimes 1 and 2 high-growth regimes and Regimes 3 and 4 low-growth regimes. Households are assumed to observe the history of the model variables $\left(k_{t}, c_{t}\right.$, and $\left.z_{t}\right)$ and that of the TFP shocks $\left(\varepsilon_{t}\right)$. Therefore, households can establish whether the economy is in the high-growth block or in the low-growth block.

We introduce the stationary variables $\mu_{t} \equiv \ln \left(z_{t} / z_{t-1}\right), \widetilde{c}_{t} \equiv c_{t} / z_{t}^{1 /(1-\alpha)}, \widetilde{k}_{t} \equiv k_{t} / z_{t}^{1 /(1-\alpha)}$ and, following Schorfheide (2005) and Liu et al. (2011), we define the steady state as the stationary equilibrium in which all shocks are shut down, including the regime shocks to the growth rate of TFP. We then derive a log-linear approximation to the equilibrium equations around the steady-state equilibrium for these stationary variables. The log-linearized Euler equation reads 5

$$
\widehat{c}_{t}=\widetilde{E}_{t} \widehat{c}_{t+1}-(\alpha-1)\left(1+(\delta-1) \beta \bar{M}^{\frac{1}{\alpha-1}}\right) \widehat{k}_{t}-\left(\frac{1}{\alpha-1}+\beta \bar{M}^{\frac{1}{\alpha-1}}(\delta-1)+1\right) \widetilde{E}_{t} \widehat{\mu}_{t+1}
$$

where $\bar{M} \equiv \exp (\bar{\mu}), \bar{\mu} \equiv\left(\bar{p}_{1}+\bar{p}_{2}\right) \mu_{H}+\left(\bar{p}_{3}+\bar{p}_{4}\right) \mu_{L}$ being the ergodic mean of the log growth rate of the economy, and $\bar{p}_{i}$ stands for the ergodic probability of being in regime $i, \widehat{\mu}_{t}, \widehat{c}_{t}$ and $\widehat{k}_{t}$ denote log-deviations of the stationary TFP growth, consumption, and capital, respectively, from their steady-state value, and $\widehat{\mu}\left(\xi_{t}\right) \equiv \mu_{t}\left(\xi_{t}\right)-\bar{\mu}$ is the log-deviation of TFP drift from its ergodic mean $\bar{\mu}$. The resource constraint is

$$
c_{s s} \widehat{c}_{t}+k_{s s} \widehat{k}_{t}=\left(\bar{M}^{\frac{\alpha}{\alpha-1}} k_{s s}^{\alpha} \frac{\alpha}{\alpha-1}+\frac{1-\delta}{\alpha-1} \bar{M}^{\frac{1}{\alpha-1}} k_{s s}\right) \widehat{\mu}_{t}+\left(\bar{M}^{\frac{\alpha}{\alpha-1}} k_{s s}^{\alpha} \alpha+(1-\delta) \bar{\mu}^{\frac{1}{\alpha-1}} k_{s s}\right) \widehat{k}_{t-1}
$$

Finally, the log-deviation of the growth rate of TFP from its ergodic level follows

$$
\widehat{\mu}_{t}=\widehat{\mu}\left(\xi_{t}\right)+\sigma_{z} \varepsilon_{t}
$$

As is standard for any RBC model, households adjust capital so as to smooth consumption intertemporally. The occurrence of TFP shocks and the succession of low-growth and high-growth regimes challenge households' ability to smooth consumption over time. When the economy is in the high-growth regime, households expect that, with some probability, the economy will enter into the low-growth regime in the future, making it harder to raise future consumption. Therefore, ceteris paribus agents raise capital today so as to raise future expected consumption $\widetilde{E}_{t} \widehat{c}_{t+1}$ vis-a-vis current consumption $\widehat{c}_{t}$. When the economy is in the low-growth regime, agents expect that, with some probability, the economy will enter into the high-growth regime in the future, making it easier to raise future consumption. Therefore, ceteris paribus agents reduce capital today so as to raise current consumption $\widehat{c}_{t}$ vis-a-vis expected future consumption $\widetilde{E}_{t} \widehat{c}_{t+1}$.

Clearly, the persistence of the regime in place critically affects consumption and capital decisions. When the current regime is expected to be short lasting, households generally adjust capital more ag-

\footnotetext{
${ }^{5} \mathrm{~A}$ detailed derivation of the steady-state equilibrium for the stationary variables and the log-linearized equations is provided in Appendix D
} 
gressively than when it is expected to be long lasting, because they deem that a switch in the next period is more likely. In contrast, households do not adjust capital so aggressively if they expect the regime to be very long lasting. When households expect that low growth or high growth has become a structural characteristic of the environment, they understand that consumption cannot be effectively smoothed out over time by adjusting capital. Thus, very persistent regimes are mostly characterized by structural changes in the level of consumption.

Given that households have limited information, the log-linearized Model (18)- 20 cannot be solved using the existing techniques that are used to solve Markov-switching models with perfect information. However, we proceed as described in the previous sections, by introducing a new set of regimes that capture the evolution of the representative household's beliefs over time. It is important to notice that in the RBC model described above, regime changes enter additively. In other words, they only affect the vector of constants $\Gamma_{c}(\cdot)$ in the canonical forms (7) or (12). In this case, the state space can be augmented with a series of dummy variables as in Schorfheide (2005), Liu et al. (2011), and Bianchi et al. (2014) and the models under imperfect information can be easily solved using standard solution methods for DSGE model, such as gensys (Sims 2002) and Blanchard and Kahn (1980). When regime changes enter multiplicatively, the matrices $\Gamma_{0}$ and $\Gamma_{1}$ are also affected. In this case, the model can be solved with any of the solution methods that have been developed for MS-DSGE models. Bianchi and Melosi (2014,

$2015)$ consider these cases and solve the model using the algorithm developed by Farmer et al. (2009).

In what follows, we adopt a standard calibration of the RBC model. We set capital's share parameter $\alpha$ to equal 0.33. The discount factor $\beta$ is equal to 0.9976 and the parameter for the physical depreciation of capital is set to equal 0.0250. The standard deviation of the TFP shock $\sigma$ is set to 0.007. We set the growth rate of TFP in the high-growth state to equal the annualized rate of $4 \%: \mu_{H}=.01$. We assume that under low-growth, the growth rate of TFP is simply zero: $\mu_{L}=0$. Furthermore, we consider several parameterizations of the transition matrix $\mathcal{P}$, allowing us to illustrate a number of different model economies that can be potentially used to address a large set of empirical issues. In actual applications, the parameterization of the transition will depend on the data and the associated empirical moments the researcher is interested to match. In this paper, we do not address any specific empirical issue as our objective is to show the scope of application of the methodology we propose.

Section 3.1 studies an economy in which agents have to learn about the likely persistence of the observed TFP growth. In Section 3.2, we consider an economy that goes through two types of phases over time: a high-growth phase that is mostly characterized by long-lasting high-growth periods with rare short-lasting low-growth periods and a low-growth phase that is mostly characterized by persistent periods of low-growth and high-growth periods of rather short duration.

\subsection{Learning the Persistence of TFP Growth: The Static-Prior Case}

In what follows, we consider an RBC model as described by equations (18)- 20 in which the economy fluctuates repeatedly between short-lasting periods of high growth and short-lasting periods of low growth. However, once in a while a prolonged phase of high growth or low growth may occur. Agents are able to infer what the growth rate is today, but they are uncertain about its likely duration.

Let us assume that the persistence of the short-lasting regimes is the same in the two blocks: $p_{11}=$ 
$p_{33}=0.5$. Analogously, we set the probabilities of staying in the long-lasting regimes so that $p_{22}=p_{44}=$ 0.95. For simplicity we assume that regimes belonging to the same block do not communicate with each other; that is, $p_{12}=p_{21}=p_{34}=p_{43}=0$. We will be more general later on. Furthermore, the transition matrix implies that once a switch to a new block occurs, agents always attach a $95 \%$ probability to being in the short-lasting regime:

$$
\begin{aligned}
& \frac{p_{31}}{p_{31}+p_{32}}=\frac{p_{41}}{p_{41}+p_{42}}=0.95 \\
& \frac{p_{13}}{p_{13}+p_{14}}=\frac{p_{23}}{p_{23}+p_{24}}=0.95
\end{aligned}
$$

Notice that conditions (21)-22 imply static prior beliefs: agents always enter the high-growth block and the low-growth block with the same beliefs. In summary, we work with the following transition matrix:

$$
\mathcal{P}=\left[\begin{array}{cc|cc}
0.50 & 0 & 0.475 & 0.025 \\
0 & 0.95 & 0.0475 & 0.0025 \\
\hline 0.475 & 0.025 & 0.50 & 0 \\
0.0475 & 0.0025 & 0 & 0.95
\end{array}\right]
$$

To illustrate the consequences of fluctuations in agents' beliefs, we simulate the economy assuming a typical path for the regimes and setting all Gaussian shocks $\varepsilon_{t}$ to zero. We assume that consumption and capital are initialized at their steady-state values. The results are reported in Figure 1. In each panel, the gray and white areas correspond to periods of low and high growth, respectively. Short-lasting regimes last for their typical duration of 2 quarters. Long-lasting regimes last for their typical duration of 20 quarters. The two right graphs report the evolution of consumption and capital in the model with learning compared to the model with perfect information in which agents can observe the current regime. The panel in the upper-left corner shows the evolution of agents' beliefs about being in the long-lasting high-growth regime and in the long-lasting low-growth regime. The panel in the lower-left corner reports the evolution of expected average TFP growth at 4-, 8-, 20-, and 40- quarter horizons. Notice that this is a convenient measure of agents' optimism/pessimism that takes into account uncertainty about the regime in place today and the possibility of regime changes.

Three features of Figure 1 deserve to be emphasized. First, right after a switch to a new block, agents believe that this switch is most likely to be short lasting. This can be seen in the top left graph when switches to new blocks occur. The reason is that agents are rational and hence are aware that regardless of whether the past regime was short lasting or long lasting, the probability of switching to the shortlasting regime in the new block is always as high as 95\%. This stems from the restrictions in 21)-(22), which imply static prior beliefs. Second, whenever a short-lasting regime is in fact realized, with the benefit of hindsight, agents' beliefs turn out to be slightly misaligned with the truth in the upper left graph because agents rationally attach a non-zero probability to being in the long-lasting regime. Third, the probability of being in the long-lasting regime smoothly increases as more realizations of the same block are observed. The top left graph shows that the probability of being in the long-lasting regime rises monotonically with the number of consecutive realizations of a particular growth rate. For instance, 

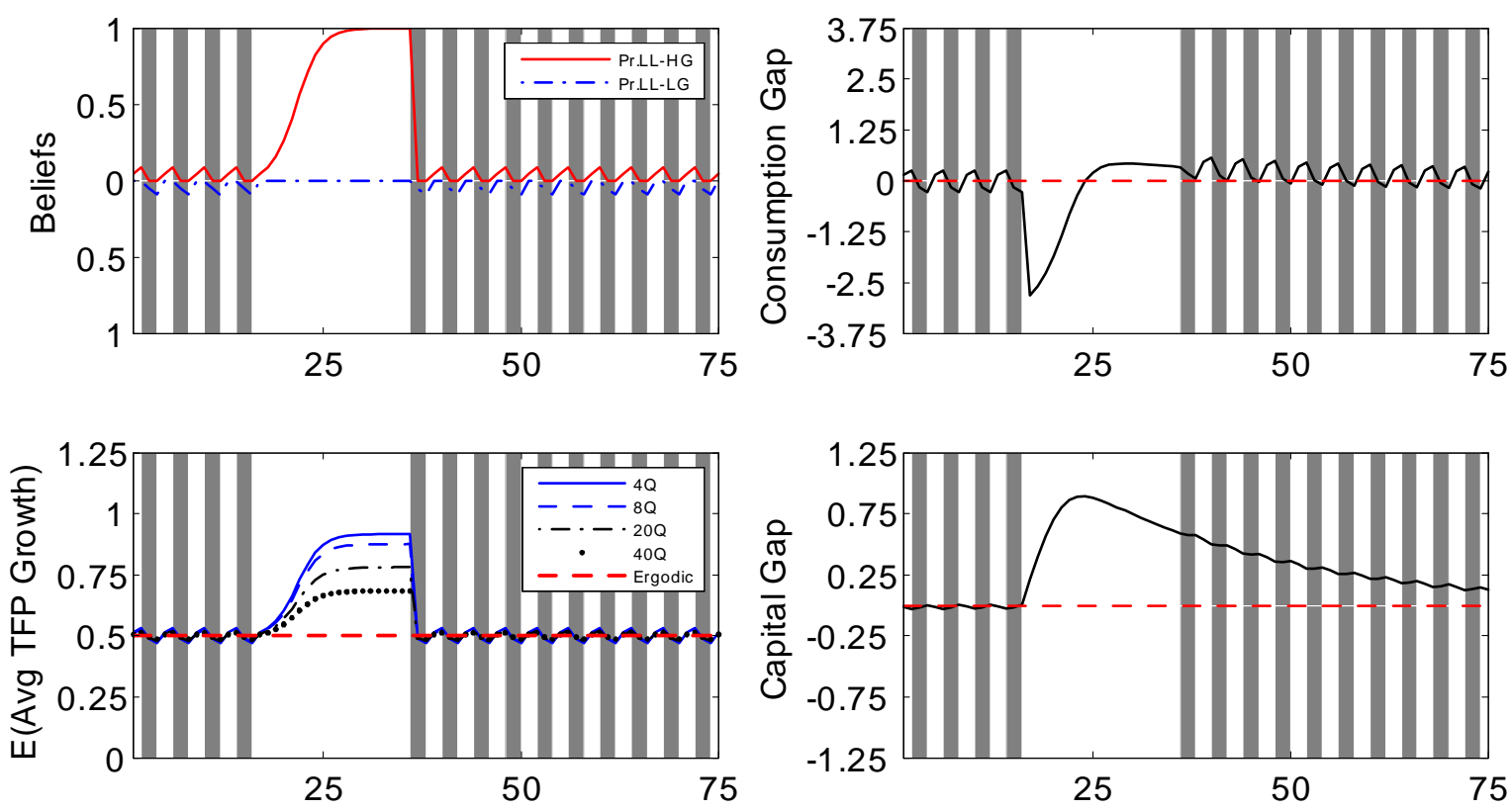

Figure 1: Beliefs and Allocations. Top left graph: Evolution of beliefs of being in the long-lasting high-growth regime (red solid line) and in the long-lasting low-growth regime (blue dashed line). Top right graph: Log-deviations of consumption from the perfect-information benchmark. Bottom left graph: Expected average growth rate of technology (annualized percentage) at various horizons. Bottom right graph: Log-deviations of capital from the perfect-information benchmark. In all graphs, gray areas denote periods of low growth.

from $t=17$ to $t=36$, the economy is in a long-lasting high-growth regime. While agents initially attach a small probability to being in the long-lasting regime, they become fully convinced after 12 consecutive periods of high TFP growth. The dynamics of beliefs is specular in a long-lasting low-growth period of typical length.

Furthermore, Figure 1 shows the evolution of optimism and pessimism and the associated dynamics of the consumption gap and the capital gap, which are defined as the log-deviation of consumption and capital from their corresponding levels under perfect information. When the economy enters the longlasting high-growth period imperfectly informed agents are not very optimistic about the duration of the high-growth regime. This is reflected in their expectations about the average growth rate of TFP that barely moves in the bottom left graph. Given that they expect that the high-growth period will be short lasting, they decide to accumulate more capital so as to smooth consumption. In contrast, if agents knew the actual realization of the high-growth regime, they would have adjusted their stock of capital less aggressively and consequently consumption would have risen more dramatically. This is why in Figure 1 we observe a negative consumption gap and a positive capital gap when the economy enters a period of long-lasting high growth. The dynamics of these gaps is specular in a long-lasting low-growth period of typical length.

As the period of high-growth consolidates, imperfectly informed agents update their beliefs until they eventually become convinced that they are in the long-lasting regime. This happens in roughly 12 quarters after the switch. As illustrated in the bottom left graph, such slow-moving beliefs cause the expected average growth rate of TFP over the next few years to adjust sluggishly. This eventually determines an 


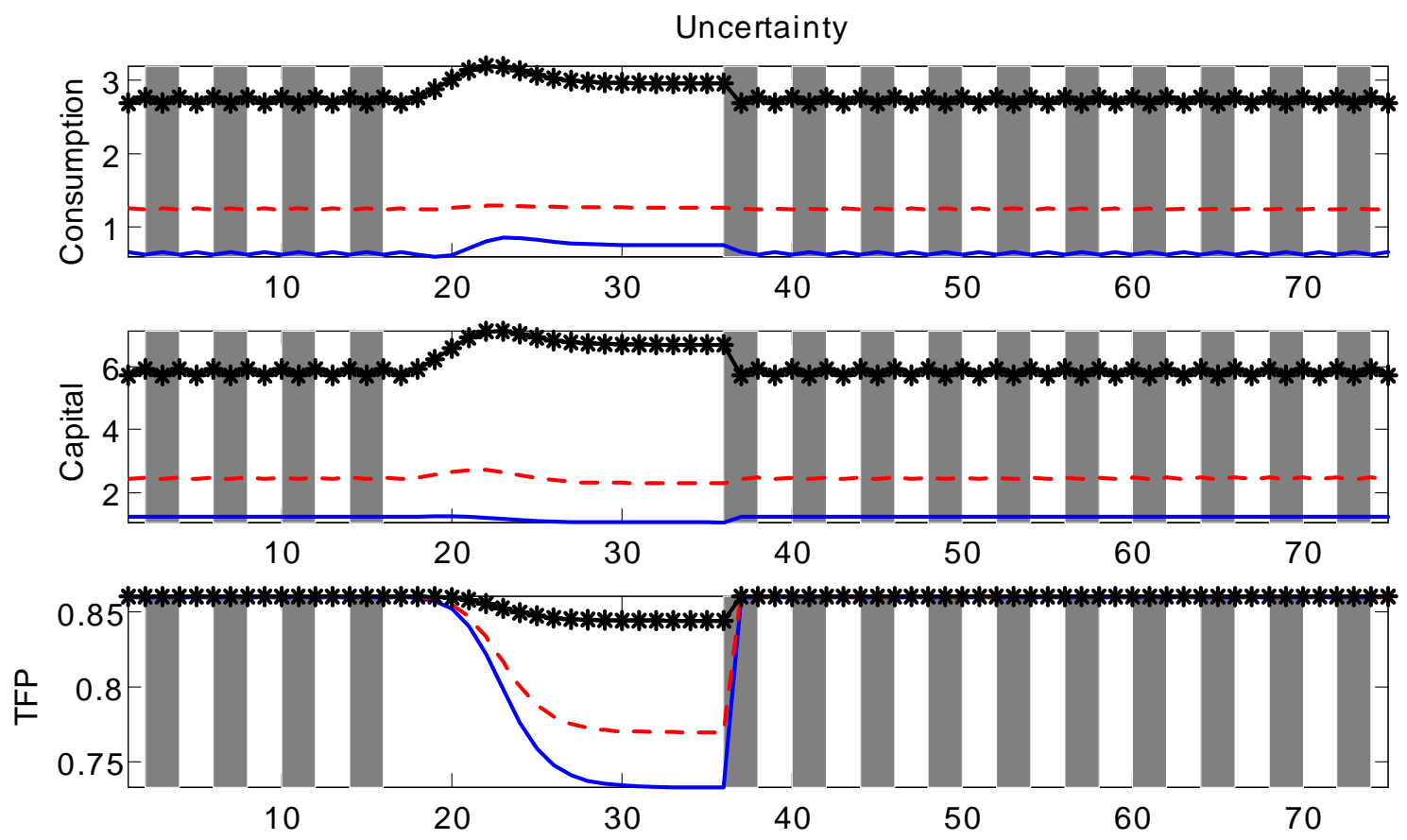

Figure 2: Evolution of Uncertainty about Consumption, Capital, and TFP Growth. The horizons are one-quarter ahead (solid line), four-year ahead (dashed line), and twenty-year ahead (circular markers). Uncertainty at an horizon $h$ is measured as the standard deviation of the variable of interest in period $t+h$ conditional on agents' information set at time $t, I_{t}$.

adjustment in the path for consumption and the consumption gap slowly fades away. Interestingly, at the end of the long-lasting high-growth period, the consumption gap becomes positive. The reason is that the sluggish evolution of optimism prompted households to accumulate capital rapidly at the beginning of the period of high growth. The relatively bigger capital stock boosts consumption as households become more optimistic about the likely duration of the high-growth period, leading to a positive consumption gap. A specular pattern characterizes the economy the moment it enters the long-lasting low-growth period.

As pointed out before, even when the economy repeatedly alternates between short-lasting periods, agents' beliefs are slightly misaligned with the truth. Let us focus on the first 16 quarters during which a sequence of short-lasting regimes are realized. While the economy is in the short-lasting high-growth regime, imperfectly informed households consume more and accumulate less capital than in the case of perfect information. The reason is that imperfectly informed agents attach some non-negligible albeit small - probability to being in the long-lasting regime. By the same token, when the economy is going through a short-lasting period of low growth, imperfectly informed households consume less and accumulate more capital than under perfect information.

Figure 2 shows the evolution of uncertainty about consumption, capital, and TFP growth rate. Uncertainty at horizon $h$ of one, four, and twenty quarters is measured using the standard deviation of the variable of interest at time $t+h$ conditional on agents' information set at time $t, \mathcal{I}_{t}$. It is worth emphasizing that this measure of uncertainty is computed taking into account the possibility of regime changes and the evolution of agents' beliefs, using the methods described in Bianchi (2013a). First, it 
should be observed that when agents are mostly convinced to be in the short-lasting high-growth regime (i.e., in the narrow white areas or at the beginning of the broad white areas), uncertainty is generally higher and remarkably similar at all horizons. Second, uncertainty about future TFP growth falls at all horizons as agents become more convinced to be in the long-lasting regime. This happens when the economy goes through a long-lasting period of high-growth, captured by the large white, in Figure 2. These two findings are not surprising, since expecting a shorter (longer) duration of the current block raises (reduces) uncertainty about future TFP developments. Furthermore, when long-lasting regimes occur, long-horizon uncertainty falls less dramatically than short-horizon uncertainty. The reason is that as agents become more convinced to be in a long-lasting regime, they deem a switch to a short-lasting regime as relatively more likely at longer horizons ${ }^{6}$

As far as the dynamics of uncertainty about consumption and capital (the highest and middle panels), it is important to notice that the main source of uncertainty about future allocations is due to the need of establishing whether and when big adjustments in allocations will occur. As shown in the right panel of Figure 1, such large adjustments are observed when long-lasting regimes occur because beliefs adjust only sluggishly to the truth. Long-horizon uncertainty about future allocations appears to be always higher than short-horizon uncertainty, because agents know that such large adjustments are always more likely to happen as the considered horizon gets longer and longer. Quite interestingly, we observe that mediumand long-horizon uncertainty follow a hump-shaped pattern during a typical long-lasting regime. On the one hand, uncertainty rapidly rises as agents gets more and more convinced of being in a long-lasting regime that is associated with a large adjustment in allocations. On the other hand, as agents become more convinced to be in the long-lasting regime, their uncertainty about future allocations falls because they become less uncertain about future TFP growth. See the lowest graph of Figure 2 .

The case studied in this section is based on a symmetric transition matrix implying that the stochastic properties of the short-lasting and the long-lasting regimes are exactly the same across blocks. Changing the relative persistence of the regimes within a block critically affects how quickly agents learn about the persistence of the regime in place. For instance, as the persistence of the two high-growth regimes become more similar, agents have to observe a longer spell of high growth to become convinced that the economy is experiencing a period of persistent high growth. To see this note that persistence is the only characteristic that make the two regimes within a block distinguishable to agents.

\subsection{A Two-Phase RBC Model}

We will now model an economy that goes through two types of phases over time: a high-growth phase that is mostly characterized by long-lasting high-growth periods with rare short-lasting low-growth periods and a low-growth phase that is mostly characterized by persistent periods of low-growth and high-growth periods of rather short duration. In such an economy waves of optimism and pessimism will spur from the past realizations of TFP growth. Therefore, we need to keep track of agents beliefs before a change in growth. This corresponds to the case of dynamic priors. In what follows, we study three RBC economies featuring low- and high-growth phases. In Subsection 3.2.1, switches to a new phase are always preceded

\footnotetext{
${ }^{6}$ Recall that the parameterization of the transition matrix $\mathcal{P}$ implies that the long-lasting regimes of both blocks are always followed by short-lasting regimes, which are associated with relatively higher uncertainty.
} 

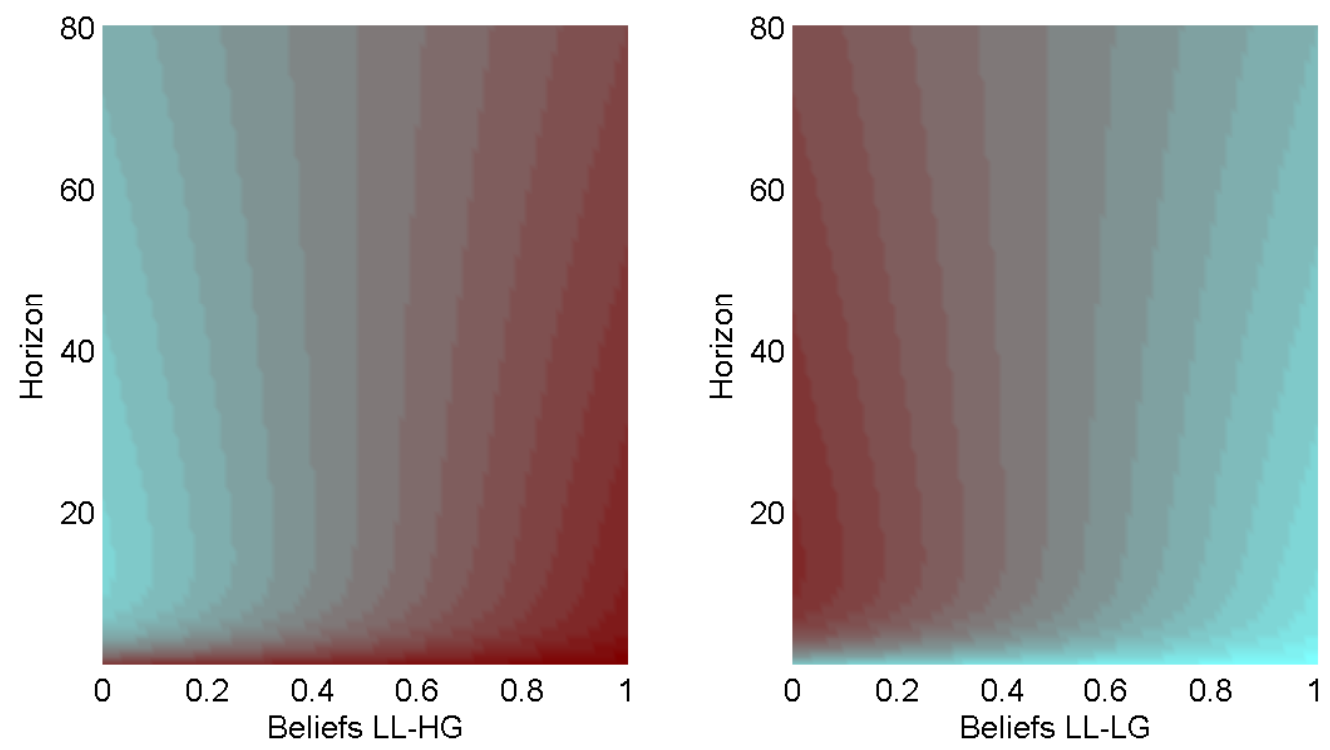

Figure 3: Expected Growth Rate of Technology at Various Horizons as a Function of Beliefs. Lighter blue areas capture expected rates that are lower than the ergodic rate. Darker red areas capture expected rates that are higher than the ergodic rate. The horizontal axes report beliefs about being in the long-lasting high-growth regime (LL-HG) and beliefs about being in the long-lasting low-growth regime (LL-LG).

by a changes in TFP growth 7 In Subsection 3.2.2, we study an economy in which switches to the low-growth phase are not necessarily marked by a changes in TFP growth. Note that whether changes of phases are preceded or not by change in TFP growth is important because agents perfectly observe changes in TFP growth.

\subsubsection{Changes of Phase Preceded by a Change in Growth}

In order to model the two phases of the business cycle, we introduce the following restrictions on the parameters of the transition matrix $\mathcal{P}$ :

$$
\begin{aligned}
& \frac{p_{31}}{p_{31}+p_{32}}=0.05<\frac{p_{41}}{p_{41}+p_{42}}=0.95 \\
& \frac{p_{13}}{p_{13}+p_{14}}=0.05<\frac{p_{23}}{p_{23}+p_{24}}=0.95
\end{aligned}
$$

Furthermore, we assume that the probability of staying in the short-lasting regimes is $p_{11}=p_{33}=0.75$. We set the probabilities of staying in the long-lasting regimes so that $p_{22}=p_{44}=0.95$. We also assume that the regimes belonging to the same block do not communicate with each other: $p_{12}=p_{21}=p_{34}=$ $p_{43}=0$. This has the important implication that a change of phase is always preceded by an observable change in growth. We will relax this restriction in the next two subsections. To sum up, the transition

\footnotetext{
${ }^{7}$ Of course, this does not mean that every time that a change in growth occurs, agents immediately conclude that the phase changed. They still have to learn about the nature of the observed change in growth.
} 
matrix reads:

$$
\mathcal{P}=\left[\begin{array}{cc|cc}
0.75 & 0 & 0.0125 & 0.2375 \\
0 & 0.95 & 0.0475 & 0.0025 \\
\hline 0.0125 & 0.2375 & 0.75 & 0 \\
0.0475 & 0.0025 & 0 & 0.95
\end{array}\right]
$$

It is important to emphasize that, in this model, the fact that the economy is currently in the highgrowth or low-growth regime plays a minor role in affecting agents' beliefs. Most of the action stems from whether agents believe that the economy has been going through a high-growth phase or a low-growth phase. Figure 3 shows agents' expectations about the growth rate of TFP $\mu_{t}$ in deviations from its ergodic level $\bar{\mu}$ at different horizons and for various initial levels of probability of being in the long-lasting high-growth regime (left plot) and low-growth regime (right plot). Notice that when agents expect to be in the long-lasting high-growth (low-growth) regime, the expected growth rate of technology differs from that in the short-lasting low-growth (high-growth) regime only at very short horizons. This is because agents are rational and are aware of conditions (23)-(24), implying that short-lasting regimes are more likely to be followed by the long-lasting regime of the opposing block.

It should be noted that conditions (23)-(24) imply that agents' beliefs are dynamic in this model. Suppose that agents mostly expect to be in the long-lasting high growth period. If in the next period the economy moves to the low-growth block, agents will mainly expect to be in the short-lasting low-growth regime. In contrast if agents believe to be in the short-lasting high growth today, then a switch to the low-growth block tomorrow will lead them to believe that the low-growth period is most likely to be long-lasting.

The upper left graph of Figure 4 reports the evolution of agents' beliefs, consumption, and capital for the case of dynamic prior beliefs. We simulate a typical path for the regimes where a low-growth phase is followed by a high-growth phase. Furthermore, we initialize agents' beliefs so that agents are confident of being in a high-growth phase 8 As agents observe 4 quarters of high growth, followed by 20 quarters of low growth, agents start to fear that the economy has switched to the low-growth phase. As a result, households are less optimistic when the economy returns to the high-growth regime. When the second realization of the long-lasting low-growth regime occurs, households become immediately convinced that the long-lasting low-growth regime is in place. Symmetrically, when the economy returns to the shortlasting high-growth regime for the third time, households believe that the high-growth regime will be long-lasting with only a $6 \%$ probability. Afterwards, the economy enters the high-growth phase by going through a short-lasting low-growth regime. Households are initially very pessimistic about the persistence of this regime expecting the low-growth regime to be long lasting. It takes two realizations of the longlasting high-growth regimes to make them fully confident that the economy has shifted to the high-growth phase.

The behavior of consumption and capital during the low-growth and the high-growth phase is analyzed in the right graphs of Figure 4. We observe that at the beginning of the first short-lasting high-growth regime, which is associated with high optimism, the consumption gap is positive. The reason is that

\footnotetext{
${ }^{8}$ This can easily happen if the economy went through a typical high-growth phase in the past and agents have finally learned about this phase.
} 

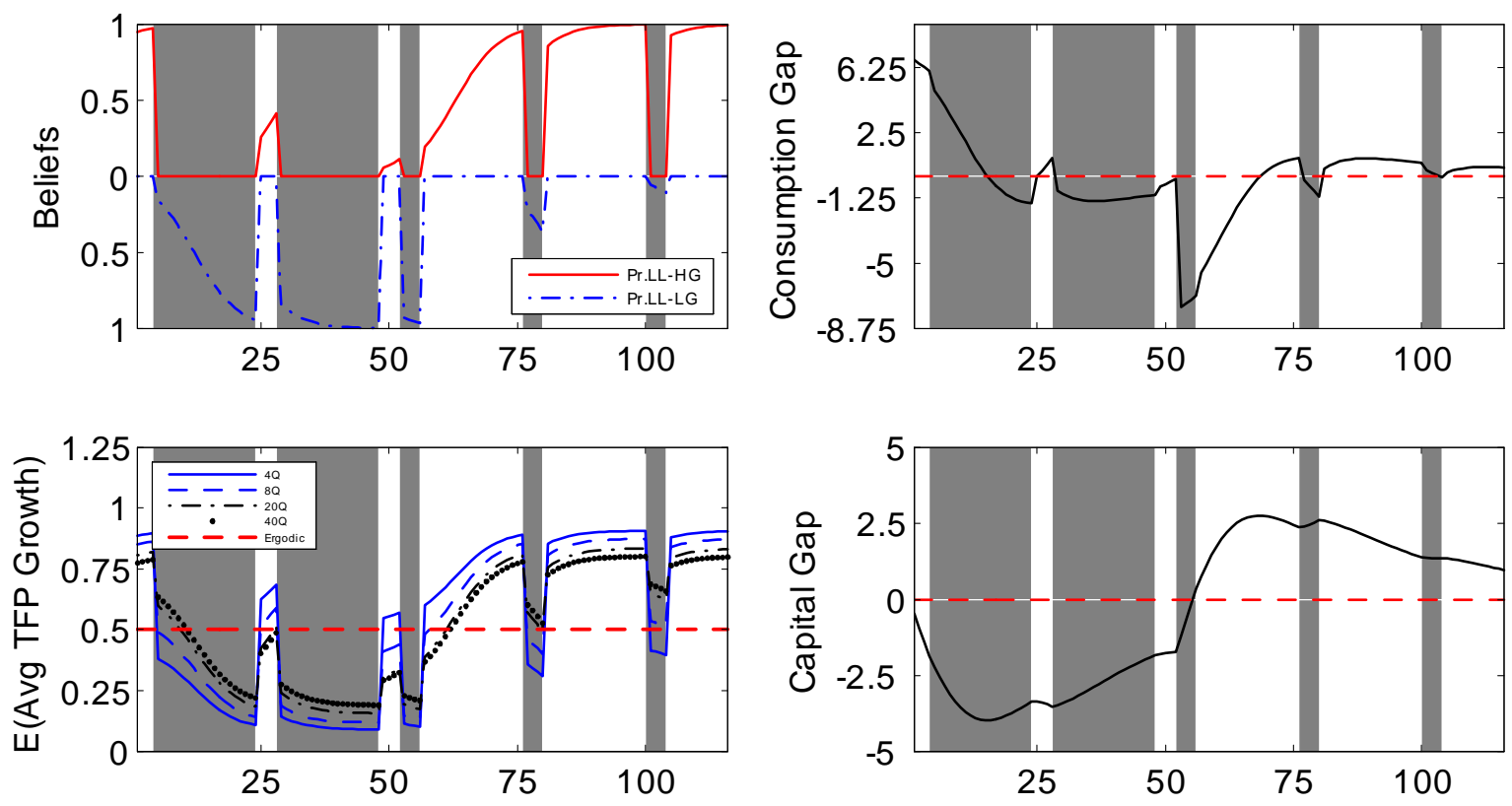

Figure 4: Beliefs and Allocations. Top left graph: Evolution of beliefs of being in the long-lasting high-growth regime (red solid line) and in the long-lasting low-growth regime (blue dashed line). Top right graph: Log-deviations of consumption from the perfect-information benchmark. Bottom left graph: Expected average growth rate of technology (annualized percentage) at various horizons. Bottom right graph: Log-deviations of capital from the perfect-information benchmark. In all graphs, gray areas denote periods of low growth.

imperfectly informed households expect this regime to be much longer lasting than what it actually turns out to be. This implies that imperfectly informed households do not raise capital as aggressively as they would if they knew that the high-growth regime is, in fact, short lasting. This leads to a negative capital gap and a positive consumption gap. When the economy enters the long-lasting low-growth regime for the first time, households mainly expect a short-lasting regime at first. As a result, households decide to cut capital fairly aggressively to sustain current consumption. Households would do otherwise, if they knew that the economy just entered the long-lasting low-growth regime. This leads to a positive consumption gap and a negative capital gap.

During the first long-lasting low-growth spell households update their beliefs until they realize that this regime is most likely long lasting, signifying that the economy must have switched to the low-growth phase. This change in agents' beliefs causes consumption and capital (the latter with some sluggishness) to become similar to the perfect-information benchmark. Interestingly, the consumption gap changes sign and becomes negative at the end of the first long-lasting low-growth spell and throughout the second low-growth period. This is due to the fact that capital adjusts sluggishly to its perfect-information level. When the second short-lasting high-growth regime occurs, agents are more convinced to have entered a low-growth phase and then optimism is smaller than in the previous high-growth period, resulting in a more contained hike in the consumption gap. The dynamics of the consumption gap and the capital gap are clearly reversed during the high-growth phase.

Figure 5 shows the evolution of uncertainty about consumption, capital, and TFP growth during the typical simulation. At time $t=1$, agents mostly expect to be in a high-growth phase that is is 


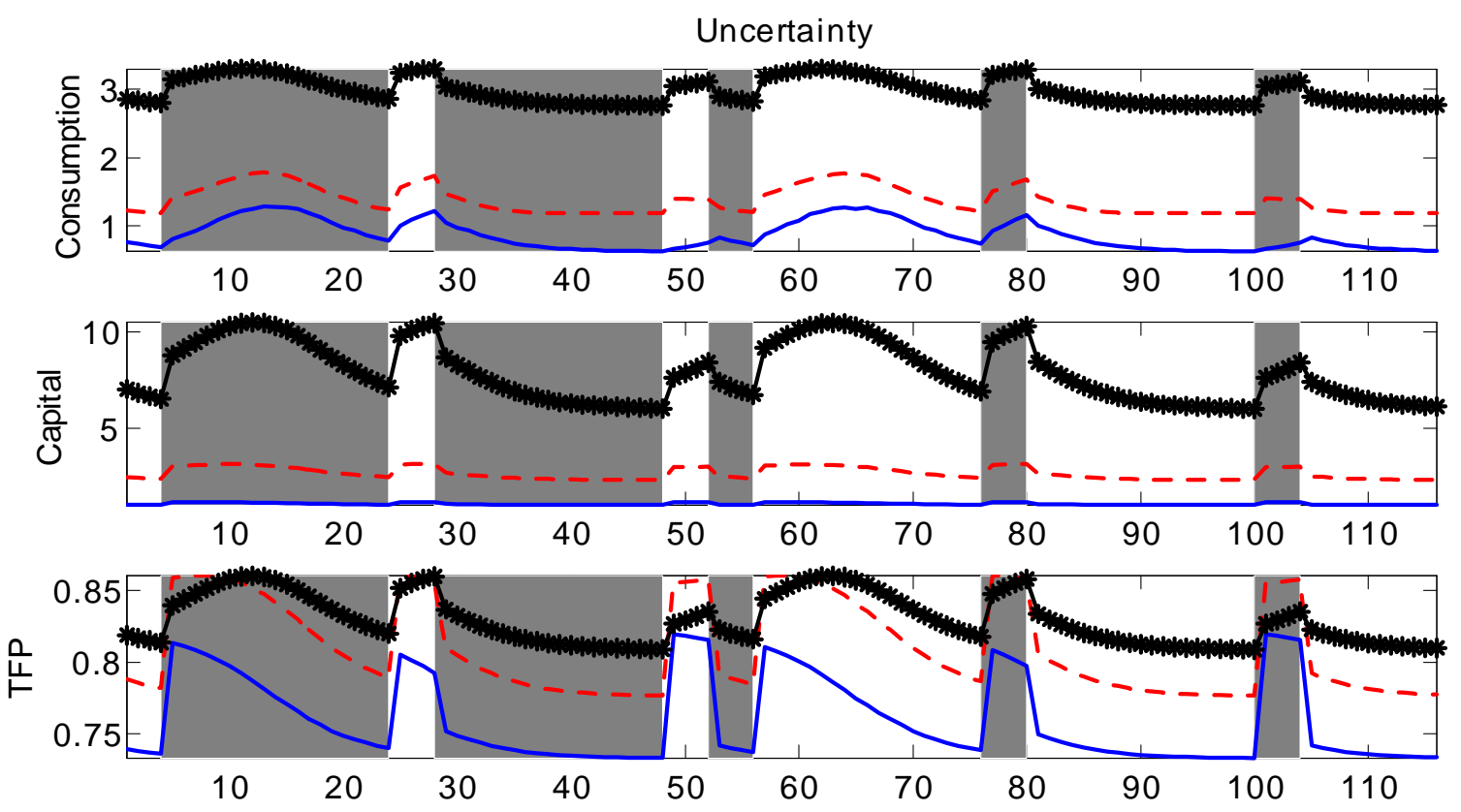

Figure 5: Evolution of Uncertainty about Consumption, Capital, and TFP Growth. The horizons are one-quarter ahead (solid line), four-year ahead (dashed line), and twenty-year ahead (circular markers). Uncertainty at an horizon $h$ is measured as the standard deviation of the variable of interest in period $t+h$ conditional on agents' information set at time $t, I_{t}$.

typically characterized by long-lasting high-growth regimes. Since agents expect to be in a long-lasting regime, their uncertainty about future TFP growth is on the low side. As the economy switches to a low-growth period at time $t=5$, agents initially expect it to be short lasting because they are still quite convinced they are living in a high-growth phase in which the typical duration of low-growth periods is relatively short. This conviction prompts agents to expect that TFP growth is likely to change shortly. Thus, their uncertainty sharply increases as the system enters the first low-growth spell. At the end of the first long-lasting low-growth period, agents have mostly learned by now that the system is going through a low-growth phase and therefore the current low-growth regime is likely to last for a fairly long period. Consequently, uncertainty about future TFP growth at all horizons fall down at the end of the long-lasting low-growth period. As agents learn that the economy is going through a low-growth phase, uncertainty soars during the short-lasting high-growth spells and falls during long-lasting low-growth periods. Specular patterns are observed in the subsequent high-growth phase.

It is worth emphasizing that due to the non-linearity implied by regime changes, uncertainty about future TFP growth is not monotonic with respect to the time horizon. In other words, agents' uncertainty does not necessarily increase with the time horizon. When agents believe that the economy is in a longlasting regime, uncertainty increases with the time horizon. If the current regime is long lasting, a switch to the short-lasting regime, which would sharply raise uncertainty about future TFP growth, is likely to occur only at long horizons. Therefore, long-horizon uncertainty is higher than the short-horizon one when agents expect the current regime to be long lasting. If instead agents believe they are in a short-lasting regime, they face relatively low uncertainty about the immediate future, but in the medium run they expect a change in TFP growth to occur, while in the long run uncertainty moves toward its 

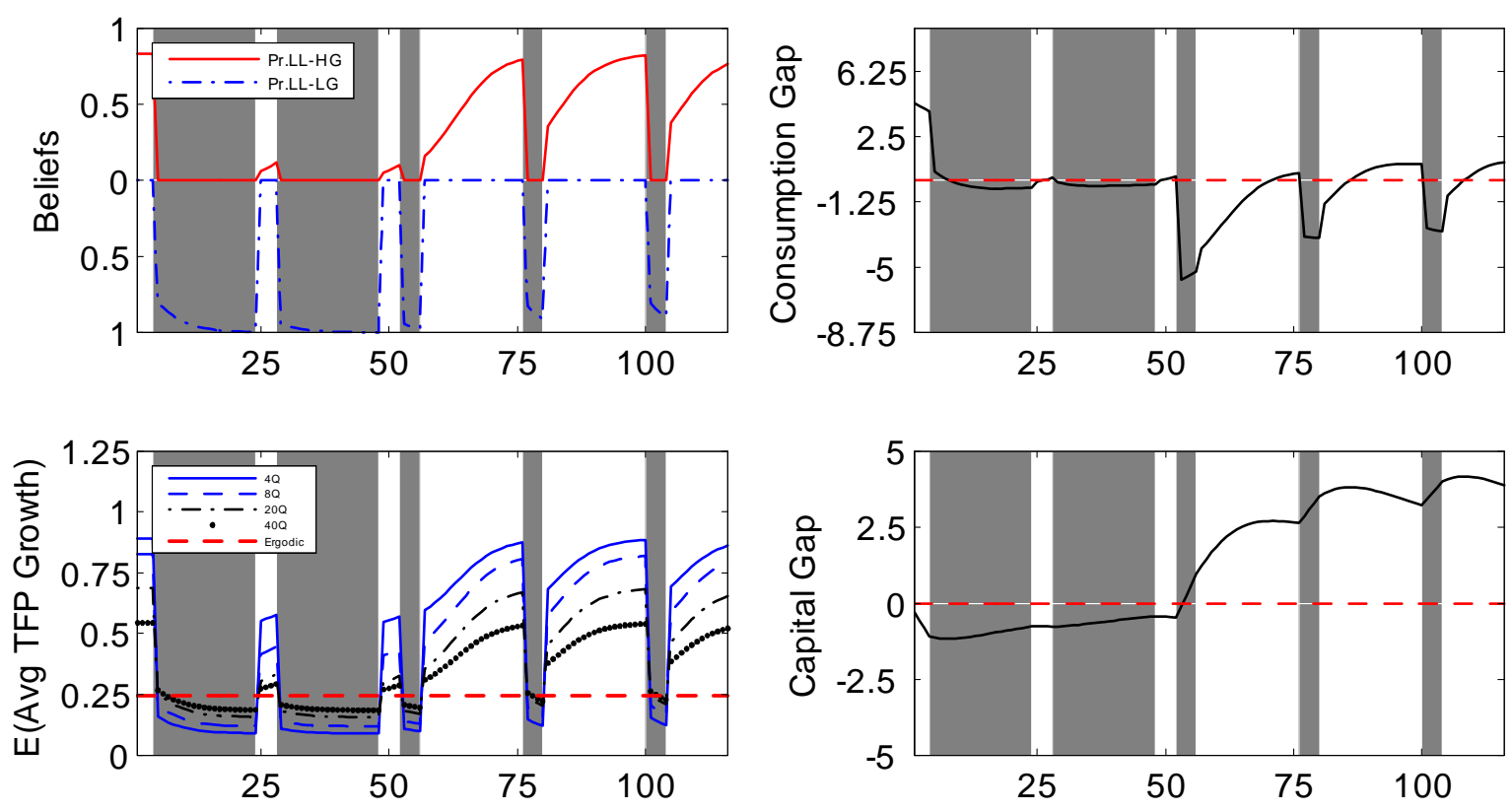

Figure 6: Beliefs and Allocations. Top left graph: Evolution of beliefs of being in the long-lasting high-growth regime (red solid line) and in the long-lasting low-growth regime (blue dashed line). Top right graph: Log-deviations of consumption from the perfect-information benchmark. Bottom left graph: Expected average growth rate of technology (annualized percentage) at various horizons. Bottom right graph: Log-deviations of capital from the perfect-information benchmark. In all graphs, gray areas denote periods of low growth.

ergodic value. This determines an hump shape in uncertainty, with medium run uncertainty higher than short run and long run uncertainty. This also implies that during the first realizations of the long-lasting regime of the two phases medium-horizon uncertainty is initially higher than long-run uncertainty. After a few periods of these long-lasting regimes, long-horizon uncertainty becomes higher than medium-horizon uncertainty. This is explained by the sharp swings in agents' beliefs occurring during the first realizations of the long-lasting regimes of the two phases. Agents enter the new phase expecting the short-lasting regime to be in place but eventually end up updating their beliefs toward the long-lasting regime. When agents attach equal odds to be in either the two regimes of the block, long-horizon uncertainty peaks whereas medium-horizon uncertainty gets lower. The fall in uncertainty about the nature of the regime in place at the end of long sequences of high or low growth causes long-horizon uncertainty to follow a hump-shaped pattern in those periods.

\subsubsection{Low-Growth Phases Not Necessarily Preceded by a Change in Growth}

We have considered so far transitions between high-growth and low-growth phases that are always marked by an observable change in TFP growth. This feature is due to the fact that so far we have assumed that the probability of switching between regimes belonging to the same block is zero. In this section we relax this assumption.

Let us use the baseline calibration and the same values for the transition matrix $\mathcal{P}$ as those used in Subsection 3.2.1, with the only exception that now the probability of switching to the short-lasting 
high-growth regime conditional on being in the long-lasting high-growth regime is nonzero. Specifically, we set $p_{21}=0.04$. The probabilities $p_{23}$ and $p_{24}$ are re-scaled so that $(24)$ is satisfied. In this context, a switch from the high-growth phase to the low-growth phase may happen without the agents observing any change in growth: The economy simply moves from the long-lasting high-growth regime to the shortlasting high-growth regime. Although the probability that such an unobserved switch would happen is quite small $\left(p_{21}=0.04\right)$, such a possibility turns out to deeply influence the dynamics of agents' beliefs and allocations.

Figure 6 reports the evolution of beliefs, average expected growth rate, consumption gap, and capital gap conditional on the same simulated path of regimes as that analyzed in the previous example. $9^{9}$ Hidden switches imply that the probability of being in the long-lasting regime never converges to one during persistent periods of high growth as agents will never become fully convinced to be in the high-growth phase.

The possibility of unobserved switches to the low-growth phase prompts households to persistently interpret short-lasting low-growth regimes as long lasting. As a result, imperfectly informed agents reduce their capital stock less aggressively than what they would have done if they knew that the economy is going through a short-lasting low-growth regime. Interestingly, high pessimism during short-lasting lowgrowth periods causes the capital gap to not exhibit mean reversion during a typical high-growth phase, leading to a sort of capital hoarding. During the low-growth phase, households learn faster that the economy is on a low-growth path compared with Figure 4. A a result, their beliefs are less misaligned with the truth and gaps are generally smaller.

Figure 7 shows the evolution of uncertainty about consumption, capital, and the TFP growth rate during the typical simulation. Three important results regarding the evolution of uncertainty about future TFP growth emerge. First, uncertainty is generally higher in the high-growth phase because the possibility of hidden switches to the low-growth phase makes the learning about the duration of the regime in place harder. Second, periods of low-growth are generally characterized by monotonically decreasing uncertainty, with long-horizon uncertainty higher than short-horizon uncertainty. Short- and mediumhorizon uncertainty is always high at the beginning of high-growth periods compared with low-growth periods. These patterns are explained by the overall rising in pessimism due to the possibility of hidden switches to the low-growth phase. As discussed earlier, when agents expect an observed regime to be long-lasting (short-lasting), short-horizon uncertainty is generally lower (higher) than long-horizon uncertainty. Since low-growth regimes are suddenly interpreted as long-lasting by agents, who become more convinced about that as the system stays in this regime, short-horizon uncertainty will be quite low and declining over time. In contrast, high-growth regimes are initially interpreted as short-lasting, implying higher uncertainty especially at short and medium horizons. Third, long-lasting high-growth regimes are characterized by hump-shaped dynamics of short-horizon uncertainty and a monotonically-increasing evolution of long-horizon uncertainty, which ends up being higher than short-horizon uncertainty. Unlike in Figure 5, long-horizon uncertainty never falls during the high-growth regimes because the possibility of hidden switches cause agents to never get fully convinced to be in the long-lasting regime. The

\footnotetext{
${ }^{9}$ To ease the comparison with the previous case with no unobservable switches, the scale of the y-axes is set to be the same as that in Figure 4 .
} 


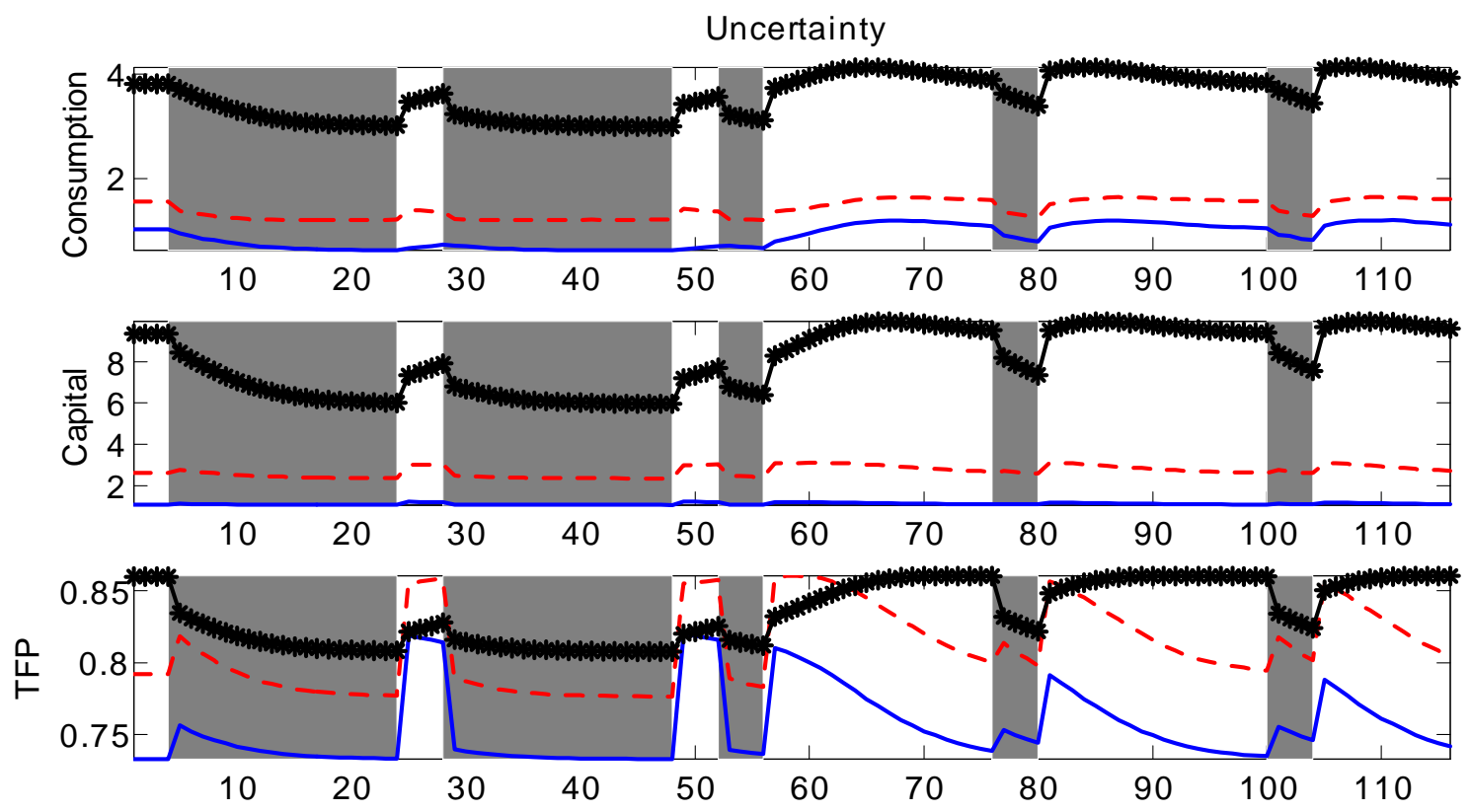

Figure 7: Evolution of Uncertainty about Consumption, Capital, and TFP Growth. The horizons are one-quarter ahead (solid line), four-year ahead (dashed line), and twenty-year ahead (circular markers). Uncertainty at an horizon $h$ is measured as the standard deviation of the variable of interest in period $t+h$ conditional on agents' information set at time $t, I_{t}$.

hump-shaped dynamics of short- and medium-horizon uncertainty during the long-lasting high-growth regimes is due to the swing in agents' beliefs. More precisely, agents initially believe to be in a shortlasting regime and eventually become convinced to be most likely in the long-lasting regime. When this happens, short-horizon uncertainty falls while long-horizon uncertainty plateaus.

\subsection{Speed and Accuracy of the Method}

While the approximation error can be shown to be extremely tiny in the model with static prior studied in Section 3.1, it is not obvious that the approximation error made in this application in which agents' priors are dynamic is small. When agents' priors is dynamic, the approximation error is tightly related to how fine is the grid $\mathcal{G}$ that approximates agents' beliefs, $\operatorname{prob}\left(\xi_{t} \mid \mathcal{I}_{t}\right)$. Let us consider the parameterization of the symmetric two-phase economy in Section 3.2.1 and set 100 equally spaced knots in our grid for each block. Furthermore, we add 194 knots to make the grid finer for beliefs near the convergence points for $\operatorname{prob}\left\{\xi_{t}=1 \mid \tau_{t}^{1}\right\}$ and $\operatorname{prob}\left\{\xi_{t}=3 \mid \tau_{t}^{1}\right\}$, which are zero for both blocks. After the refinement of the grid of beliefs introduced in steps 3-4 of Section 2.2.2, we are left with 213 grid points per block. Even if the number of regimes seems enormous, solving the model takes 5.23 seconds in Matlab on a 64-bit desktop endowed with an Intel core processor i7-2600 CPU at $3.40 \mathrm{GHz} 10$. Figure 8 reports the absolute forecast error, which is computed by taking the absolute difference between $\operatorname{prob}\left\{\xi_{t+h} \mid \mathcal{I}_{t}\right\}$ approximated using the matrix $\widehat{\mathcal{P}}$ and the true probability that can be easily worked out using equations (3) and (4). The forecast errors are computed using various initial beliefs of being in the short-lasting regime of a

\footnotetext{
${ }^{10}$ In the case with static prior beliefs, which was analyzed in Section 3.1 it takes 0.10 second to compute the matrix $\widehat{\mathcal{P}}$ and to solve the model with gensys.
} 

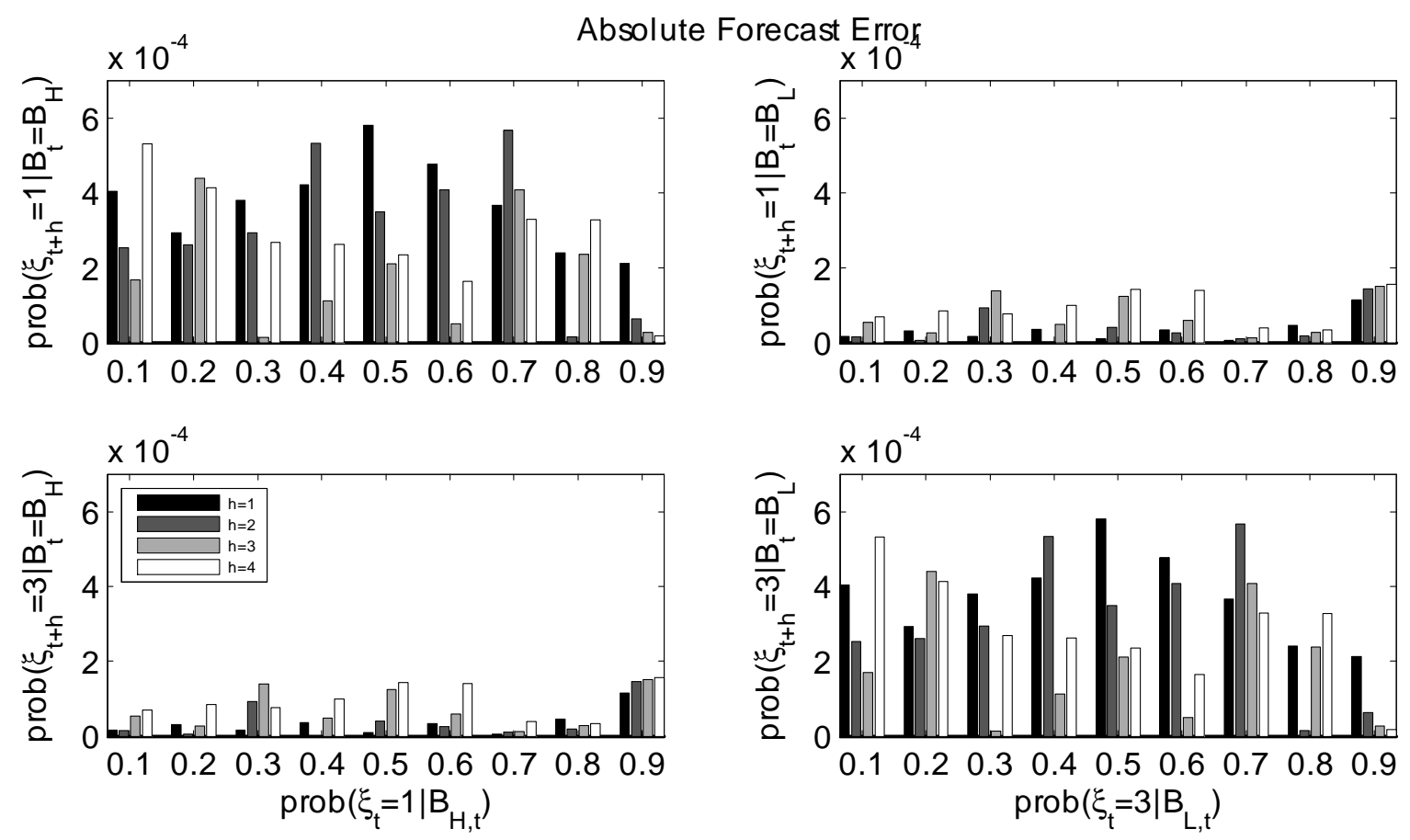

Figure 8: Absolute Forecast Errors. Left panel: Absolute forecast error about the probability of being in the short-lasting regimes $\operatorname{prob}\left\{\xi_{t+h}=1 \mid \mathcal{I}_{t}\right\}$ (upper graphs) and $\operatorname{prob}\left\{\xi_{t+h}=3 \mid \mathcal{I}_{t}\right\}$ (lower graphs), in $h=1,2,3$, and 4 quarters from period $t$ conditional on being in the high-growth block with initial beliefs $\operatorname{prob}\left\{\xi_{t}=1 \mid \mathcal{I}_{t}\right\}$, which are reported on the horizontal axes. Right panel: Absolute forecast error about the probability of being in the short-lasting regimes $\operatorname{prob}\left\{\xi_{t+h}=1 \mid \mathcal{I}_{t}\right\}$ (upper graph) and $\operatorname{prob}\left\{\xi_{t+h}=3 \mid \mathcal{I}_{t}\right\}$ (lower graph), in $h=1,2,3$, and 4 quarters from period $t$ conditional on being in the low-growth block with initial beliefs $\operatorname{prob}\left\{\xi_{t}=3 \mid \mathcal{I}_{t}\right\}$, which are reported on the horizontal axes. Different color bars denote different horizons $h$ of forecast. $B_{t}$ denotes the block in place at time $t$, which can be the high-growth one, $B_{H}$, or the low-growth one, $B_{L}$.

given block, $\operatorname{prob}\left(\xi_{t}=1 \mid \mathcal{I}_{t}\right)$ and $\operatorname{prob}\left(\xi_{t}=3 \mid \mathcal{I}_{t}\right)$, which are reported on the horizontal axes of the plots in Figure 8. The left panel refers to the situation in which the economy is initially in the high-growth block, while the right panel shows the approximation errors when the initial state of the economy is low growth. Approximation error appears to be very small at all horizons 11 Finally note that the plots are symmetric (i.e., the upper left one is identical to the lower right one and the lower left one is identical to the upper right one) because the primitive transition matrix $\mathcal{P}$ is symmetric and the initial grids for the beliefs in the two blocks are chosen to be identical.

\section{Discussion}

Summarizing, the methods outlined above show that one can recast the Markov-switching DSGE model with learning as a Markov-switching rational expectations system in which the regimes are indexed with respect to agents' beliefs. In the case of static priors, the number of consecutive realizations of a block represents a sufficient statistic to index agents' beliefs. In the case of dynamic priors, agents' beliefs

\footnotetext{
${ }^{11}$ We also checked stability of the law of motion as the number of grid points increases. The approximation turns out to be accurate even using this criterion. We report results for the absolute forecast errors because these are not model specific.
} 

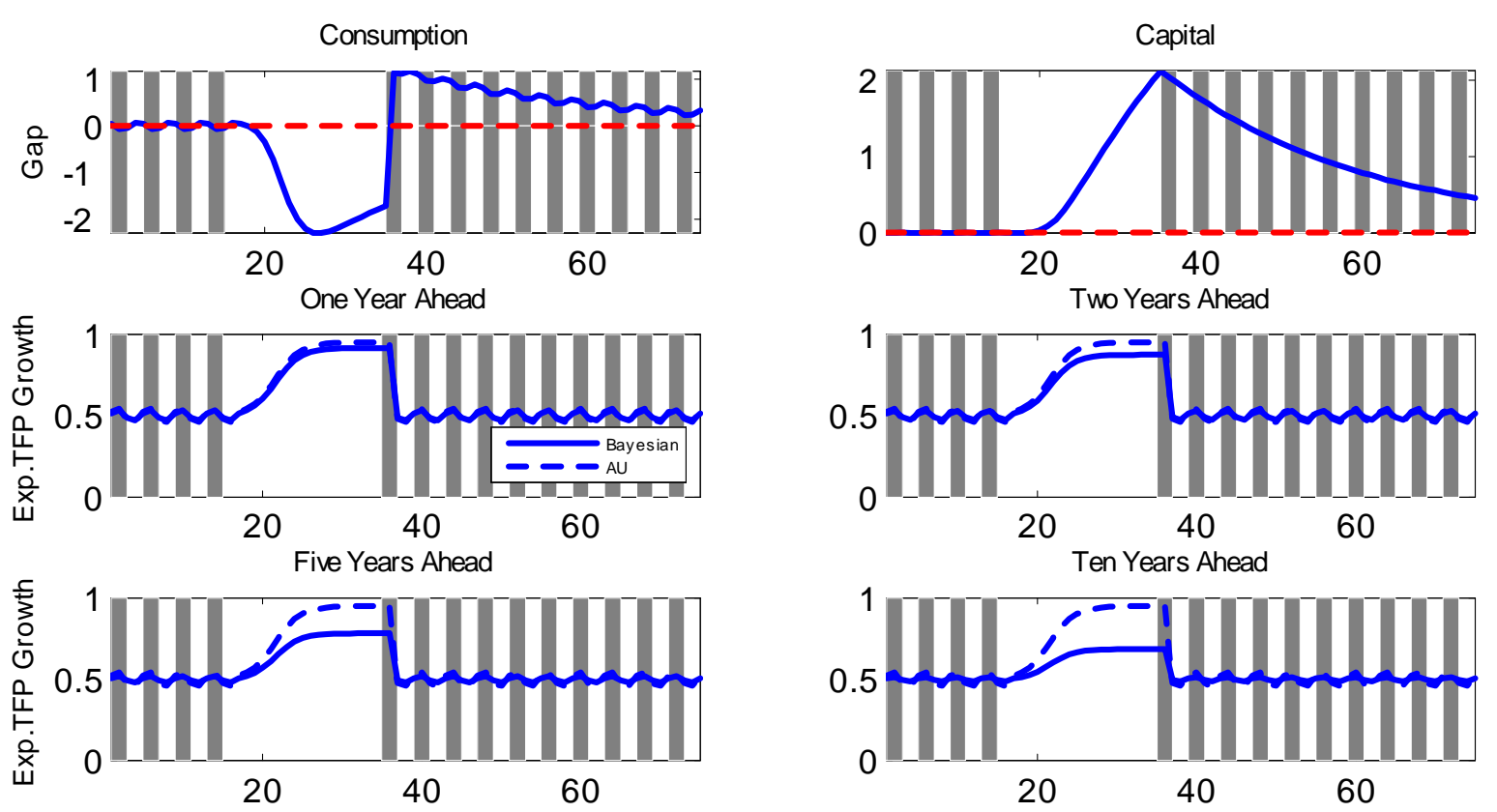

Figure 9: Full rationality vs. Anticipated Utility. The top plots show the gap of consumption and capital between the case of Rational learning and that of anticipated utility. A positive value for the gap means that consumption or capital are higher in the rational case. The middle and bottom plots report the expected TFP growth at one-, two-, five-, and ten-year horizon. The solid line denotes the case of fully rational case; the dashed line denotes the case of anticipated utility.

are mapped into a grid. In both cases, a new transition matrix that characterizes the joint evolution of agents' beliefs and model parameters is derived.

Section 4.1 highlights the main differences of our approach from the tradition learning literature. In Section 4.2, we discuss the tractability of the proposed method and assess its suitability for econometric applications. In Section 4.3, we deal with the applicability of our method to nonlinear models.

\subsection{Anticipated Utility}

It is worth emphasizing that the proposed approach allows us to easily model economies in which agents know that they do not know. In other words, agents form expectations taking into account that their beliefs will change in the future according to what they will observe in the economy. This is why the laws of motion (9) and (13) characterizing the behavior of the model depend on the current beliefs and the expanded transition matrix defining the joint evolution of agents' beliefs and model parameters. This represents a substantial difference with the anticipated utility approach in which agents form expectations without taking into account that their beliefs about the economy will change over time (e.g., Evans and Honkapohja 2001; Cogley, Matthes, and Sbordone 2011). Furthermore, the approach described above differs from the one traditionally used in the learning literature in which agents form expectations according to a reduced-form law of motion that is updated recursively using the discounted least-squares estimator (Eusepi and Preston 2011). The advantage of adaptive learning is the extreme flexibility given that, at least in principle, no restrictions need to be imposed on the type of parameter instability characterizing the model. However, such flexibility does not come without a cost, given that agents are 
not really aware of the model they live in, but only of the implied law of motion. Instead, in this paper agents fully understand the model, they are uncertain about the future, and they are aware of the fact that their beliefs will evolve over time based on what they observe.

In this section, we assess the error implied by the anticipated utility assumption in the context of the prototypical RBC model considered in our paper. Just to keep things simple we use the parameterization of Section 3.1, which is the one with static prior beliefs. We model the case of anticipated utility as follows 12 At every point in time $t$ the RBC mode ${ }^{13}$ is solved by assuming that there exist two regimes evolving according to the transition matrix $P^{A U}(t)$, where if the system has been for $s>0$ consecutive quarters in the high-growth regime

$$
\mathcal{P}^{A U}(t)=\left[\begin{array}{cc}
\operatorname{prob}\left\{\tau_{t}^{1}=s+1 \mid \tau_{t}^{1}=s\right\} & 1-\operatorname{prob}\left\{\tau_{t}^{1}=s+1 \mid \tau_{t}^{1}=s\right\} \\
1-\operatorname{prob}\left\{\tau_{t}^{2}=2 \mid \tau_{t}^{2}=1\right\} & \operatorname{prob}\left\{\tau_{t}^{2}=2 \mid \tau_{t}^{2}=1\right\}
\end{array}\right]
$$

or if the system has been for $s>0$ consecutive quarters in the low-growth regime

$$
\mathcal{P}^{A U}(t)=\left[\begin{array}{cc}
\operatorname{prob}\left\{\tau_{t}^{1}=2 \mid \tau_{t}^{1}=1\right\} & 1-\operatorname{prob}\left\{\tau_{t}^{1}=2 \mid \tau_{t}^{1}=1\right\} \\
1-\operatorname{prob}\left\{\tau_{t}^{2}=s+1 \mid \tau_{t}^{2}=s\right\} & \operatorname{prob}\left\{\tau_{t}^{2}=s+1 \mid \tau_{t}^{2}=s\right\}
\end{array}\right]
$$

where Regime 1 is the high-growth regime and Regime 2 is the low-growth regime. Recall, $\operatorname{prob}\left\{\tau_{t}^{1}=s+1 \mid \tau_{t}^{1}=s\right\}$, $\operatorname{prob}\left\{\tau_{t}^{2}=s+1 \mid \tau_{t}^{2}=s\right\}$ are defined by equation (8).

Under the assumption of anticipated utility, agents keep conducting Bayesian updating of their beliefs about the persistence of the observed growth rate of TFP. This is reflected by the fact that the probabilities $\operatorname{prob}\left\{\tau_{t}^{1}=s+1 \mid \tau_{t}^{1}=s\right\}$ and $\operatorname{prob}\left\{\tau_{t}^{2}=s+1 \mid \tau_{t}^{2}=s\right\}$ are updated by using equations (3)-(4). However, bounded rational agents do not take into account that their beliefs about the persistence of the observed period of growth will change according to what they will observe in the future. In fact, agents overlook that if in the next period $t+1$ the system is still in the high-growth regime, they should rationally expect a persistence of the ongoing regime to be $\operatorname{prob}\left\{\tau_{t}^{1}=s+2 \mid \tau_{t}^{1}=s+1\right\}$, which is different from (in most cases, it is strictly higher than) what they actually expect (i.e., $\operatorname{prob}\left\{\tau_{t}^{1}=s+1 \mid \tau_{t}^{1}=s\right\}$ ). This implies that agents tend to be overpessimistic about the growth prospects of the economy in high-growth periods. Furthermore, the matrix $P^{A U}(t)$ captures the fact that agents do not fully understand how their beliefs will evolve in case of a switch to the low-growth block. This second type of bias contributes to make agents overoptimistic about the growth prospects of the economy when the economy is going through high-growth periods. When the economy is in a low-growth regime, the specular argument applies.

We consider the parameterization used for the static-prior simulation (Section 3.1). In the middle and lower panel of Figure 9, we plot the expected TFP growth at one-, two-, five-, and ten-year horizon during the simulation exercise conducted in Section 3.1. The solid line denotes the case of fully rational case

\footnotetext{
${ }^{12}$ An alternative way to model anticipated utility in our framework is to assume that beliefs about future outcomes are equal to the current beliefs in every period. This stronger version of anticipated utility implies that bounded-rational agents are not aware of regime changes and expect that the current regime will be in place forever. This stronger version of anticipated utility leads to sizable approximation errors. We use a weaker version of anticipated utility in the paper so as to give this approach a fair chance to approximate the fully rational solution.

${ }^{13}$ To simplify the model comparison, the model is loglinearized around the same ergodic steady-state equilibrium as the $\mathrm{RBC}$ model studied in the previous sections.
} 
obtained by applying our methodology whereas the dashed line denotes the case of anticipated utility. We can observe that the assumption of anticipated utility leads to overoptimism at the end of a long-lasting high-growth period of a typical duration. The reason is that agents underestimate the duration of a period of low growth that might materialize in the future. In period of low growth the dynamics of beliefs and capital accumulation are specular.

In the top panel of Figure 9 we report the gap of consumption and capital between the fully rational case and that of anticipated utility. A positive value for the gap means that consumption or capital are lower in the anticipated-utility case compared to the fully rational case. Overly optimistic agents accumulate relatively less capital during periods of long-lasting high growth. When agents were bounded rational, capital stock would be lower than the fully rational case by slightly more than $2 \%$ at the end of the typical long-lasting high-growth period. As the economy switches to short-lasting low growth there is a big increase in capital accumulation under anticipated utility. The reason is that now agents' beliefs become very similar with those in the rational case and agents want to fill the capital gap accumulated in the past. This induces a huge drop in consumption by the bounded rational agents in the period when the economy exits a long-lasting high-growth period. Assuming anticipating utility would lead to an overestimation of this drop in consumption growth rate by about 5 percentage points. A specular pattern would arise in the aftermath of a typical long-lasting low-growth period.

Cogley and Sargent (2008) use a business cycle model with no capital accumulation to show that anticipated utility provides an accurate approximation of the solution of certainty-equivalent models with fully rational learning. The reason why we reach different conclusions in this paper has to do with the fact that our linear-quadratic model features a state variable (i.e., the physical capital) that, under standard calibration, turns out to converge to steady state fairly sluggishly. This implies that past mistakes induced by anticipated utility cannot be undone so quickly. Finally, note that similarly to Cogley and Sargent (2008) the approximation error is negligible when capital is close to its steady state value. This is the case at the beginning of the simulation where both gaps are virtually zero. Importantly, as the top right graph of Figure 9 shows, the capital gap is still non-zero after a typical succession of short-lasting regimes lasting forty quarters. Such a sluggish adjustment of the physical capital implies that any subsequent deviation to one of the two typical long-lasting regimes would exacerbate the approximation error associated with the anticipated-utility approach. This is suggestive that the unconditional approximation error associated with the anticipated-utility approach is first order.

\subsection{Likelihood Estimation}

It is also important to emphasize the extreme tractability of the approach taken in this paper. The solutions (9) and (13) can be easily combined with an observation equation and used in an estimation algorithm. For example, Bianchi and Melosi (2015) estimate a prototypical New-Keynesian DSGE model, in which agents form beliefs about the likely duration of deviations from active inflation stabilization policies. The estimation of this new class of models is possible for three main reasons. First, even if the final number of regimes can be extremely high, the model imposes very specific restrictions on the allowed regime paths and on the link between observable outcomes and agents' beliefs. This implies that when evaluating the likelihood, a relatively small number of regime paths has to be taken into account. Second, 
the statistical properties of the different regimes can vary substantially and depend on the probability of moving across regimes. Therefore, identification of the transition matrix is not only given by the frequency with which the different regimes occur, but also by the laws of motion characterizing the different regimes. Finally, the number of extra parameters with respect to a model with perfect information is very low, if not zero, while the resulting dynamics can be substantially enriched. For example, Bianchi and Melosi (2014) show that a period of fiscal distress can lead to a run-up in inflation that lasts for decades.

From a computational point of view, there might be a concern about the time required to solve the model when the final number of regimes becomes very large. This turns out not to be a problem. If regime changes enter in an additive way, affecting only the matrix $\Gamma_{c}$, the model can be solved with standard solution algorithms such as gensys (Sims 2002) or Blanchard and Kahn (1980) and the high dimensionality of the transition matrix is not found to give rise to computational hurdles. However, in many situations we might want to model regime changes that enter in a multiplicative way. For example, we might want to allow for changes in the Taylor rule parameters. In this case, the matrices $\Gamma_{0}$ and $\Gamma_{1}$ are also affected and we need to rely on solution methods developed to solve MS-DSGE models. However, according to our experience based on the use of the approach proposed by Farmer et al. (2009), even in this case a solution can be obtained in a matter of seconds because the transition matrix governing the evolution of the regimes is very sparse. Therefore, the methods described in this paper provide a promising tools for modeling information frictions, animal spirits, and shocks to agents' beliefs in a general equilibrium framework suitable for structural estimation.

\subsection{Extension to Nonlinear Models}

To illustrate our methods, we have referred to linear models of the form (1) because this is currently the most popular modeling framework when it comes to solving and estimating general equilibrium models subject to parameter instability. However, this restriction can be easily relaxed. In fact, it can be shown that all the results about the dynamics of agents' beliefs in Section 2 apply to the nonlinear case as well. To see this, one should notice that we maintain the assumption that agents fully understand the model and can observe the endogenous variables and the shocks. Therefore, at each point in time they are able to infer the block that is in place. Once the history of the realizations of the two blocks is known, the dynamics of agents' beliefs are pinned down by the properties of the transition matrix $\mathcal{P}$ that is known to agents. Therefore, the evolution of beliefs does not depend on the model under consideration or the order of approximation.

It is worth emphasizing that application of our method to nonlinear models sets a promising research agenda aimed to investigate the macroeconomic effects of swings in uncertainty due to changes in fundamentals or policy-makers' behavior. In light of this, the progresses made in efficiently estimating DSGE model through perturbation methods (e.g., Fernandez-Villaverde and Rubio-Ramirez 2006 and Foerster, Rubio-Ramirez, Waggoner, and Zha 2011) have to be regarded as complementary to this line of studies. 


\section{Concluding Remarks}

This paper has developed methods to solve general equilibrium models in which agents are subject to waves of optimism, pessimism, and uncertainty. Agents in the model are fully rational, understand the structure of the economy, and know that they do not know. Therefore, when forming expectations they take into account that their beliefs will evolve in response to realized observable economic outcomes, the behavior of other agents in the model, or both. The central insight consists of creating an expanded number of regimes indexed with respect to agents' beliefs. The resulting law of motion reflects agents' uncertainty and can be expressed in state space form. Therefore, the framework proposed in this paper is suitable for structural estimation. Another important contribution of the paper is to show the large role played by changes in agents' beliefs in shaping the predictions we draw from rational expectations models. The applications studied in this paper aim to show the scope of applicability of the proposed methods. Finally, note that we have confined our analysis to the case of a primitive transition matrix of four regimes. Nothing prevents us from constructing a larger transition matrix with a potentially richer scope for learning. The choice of the transition matrix depends on the feature of the stochastic process the researcher wishes to estimate. The results of this paper can be easily extended to more articulated transition matrices.

Adding signals to our learning mechanism can be seen as a convenient way to model shocks to beliefs; that is, exogenously driven changes in beliefs that are not associated with changes in the economy's state. While this is not the first paper to use signals as shocks to beliefs (e.g., Lorenzoni 2009, Angeletos and La'O 2010 and 2013) the approach proposed in this paper has the important advantage of keeping the model very tractable. This feature makes our methods potentially suitable for studying shocks to beliefs in likelihood-based estimated large-scale DSGE models (e.g., Christiano, Eichenbaum, and Evans 2005 and Smets and Wouters 2007). Combined with the methods developed in Bianchi (2013a), we can also study the effects of shocks to beliefs on uncertainty.

As scholars develop methods to efficiently solve and estimate DSGE models through high-order perturbation (e.g., Fernandez-Villaverde and Rubio-Ramirez 2006 and Foerster, Rubio-Ramirez, Waggoner, and Zha 2011), the methods developed in this paper lay down a convenient framework for investigating the effects of changes in economic fundamentals or animal spirits on uncertainty and the feedback effects of such swings in uncertainty on the economic dynamics.

\section{Appendix}

The appendices are organized as follows. Appendix A works out the recursions (3) and (4) that pin down the dynamics of beliefs within blocks. Appendix $\mathrm{B}$ proves Proposition 1 that ensures the convergence of the difference equations (3)-(4). Appendix $\mathrm{C}$ details the algorithm to construct the transition matrix

$\widehat{\mathcal{P}}$ when agents receive signals. Appendix $\mathrm{D}$ characterizes the steady-state equilibrium for stationary variables in the $\mathrm{RBC}$ model and obtains the log-linearized equations of this model.

Note that the convergence results, which are proven in Appendices B, could be derived by working on the submatrices of each block. However, we have decided to work with the solution of the difference equations (3) and (4) because this approach is familiar to a wider audience. 


\section{A Deriving the Law of Motion for Beliefs}

In this appendix, we want to show two propositions.

Proposition 2 The rational difference equations (3) and (4) hold true

Proof. Recall that equation (3) describes the dynamics of beliefs within Block 1. Consequently, this equation holds when $\tau_{t}^{1}>1$. The Bayes' theorem can be applied to characterize the probability of being in Regime 1 given that the system is in Block $1\left(\tau_{t}^{1}>1\right)$ :

$$
\operatorname{prob}\left(\xi_{t}=1 \mid \tau_{t}^{1}\right)=\frac{p\left(\tau_{t}^{1}=\tau_{t-1}^{1}+1 \mid \xi_{t}=1\right) p\left(\xi_{t}=1 \mid \tau_{t-1}^{1}\right)}{\sum_{i=1}^{4} p\left(\tau_{t}^{1}=\tau_{t-1}^{1}+1 \mid \xi_{t}=i\right) p\left(\xi_{t}=i \mid \tau_{t-1}^{1}\right)}
$$

But if $\tau_{t}^{1}=\tau_{t-1}^{1}+1$, then the likelihood is such that

$$
p\left(\tau_{t}^{1}=\tau_{t-1}^{1}+1 \mid \xi_{t}=1\right)=p\left(\tau_{t}^{1}=\tau_{t-1}^{1}+1 \mid \xi_{t}=2\right)>0
$$

and

$$
p\left(\tau_{t}^{1}=\tau_{t-1}^{1}+1 \mid \xi_{t}=3\right)=p\left(\tau_{t}^{1}=\tau_{t-1}^{1}+1 \mid \xi_{t}=4\right)=0
$$

The equality in the first expression reflects the fact that agents cannot distinguish regimes belonging to the same block. The inequality sign in the first expression and the equality sign in the second expression are due to the fact that the system is in Block 1 at time $t$, ruling out the possibility that either Regime 3 or Regime 4 is realized. These results allow us to write:

$$
\operatorname{prob}\left(\xi_{t}=1 \mid \tau_{t}^{1}\right)=\frac{p\left(\xi_{t}=1 \mid \tau_{t-1}^{1}\right)}{\sum_{i=1}^{2} p\left(\xi_{t}=i \mid \tau_{t-1}^{1}\right)}
$$

Since $p\left(\xi_{t}=i \mid \tau_{t-1}^{1}\right)=\sum_{j=1}^{2} p\left(\xi_{t-1}=j \mid \tau_{t-1}^{1}\right) p_{j i}$, then

$$
\operatorname{prob}\left(\xi_{t}=1 \mid \tau_{t}^{1}\right)=\frac{\sum_{j=1}^{2} p\left(\xi_{t-1}=j \mid \tau_{t-1}^{1}\right) p_{j 1}}{\sum_{i=1}^{2} \sum_{j=1}^{2} p\left(\xi_{t-1}=j \mid \tau_{t-1}^{1}\right) p_{j i}}
$$

Furthermore, note that $p\left(\xi_{t-1}=2 \mid \tau_{t-1}^{1}\right)=1-p\left(\xi_{t-1}=1 \mid \tau_{t-1}^{1}\right)$ and after straightforward manipulations leads to equation (3). Equation (4) can be proved analogously.

\section{B Proof of Proposition 1}

We will characterize the convergence of $\operatorname{prob}\left(\xi_{t}=1 \mid \tau_{t}^{1}\right)$ as the number of consecutive periods spent in Block 1, $\tau_{t}^{1}$, grows large. We will denote $\lim _{\tau_{t}^{1} \rightarrow \infty} \operatorname{prob}\left(\xi_{t}=1 \mid \tau_{t}^{1}\right)=x$ using $\operatorname{prob}\left(\xi_{t}=1 \mid \tau_{t}^{1}\right) \longrightarrow x$ and the 
characteristic roots of equation (3) with :

$$
\begin{aligned}
& \widetilde{\lambda}_{1} \equiv \frac{p_{11}-p_{22}-2 p_{21}-\sqrt{\left(p_{11}-p_{22}\right)^{2}+4 p_{21} p_{12}}}{2\left(p_{11}+p_{12}-p_{21}-p_{22}\right)} \\
& \widetilde{\lambda}_{2} \equiv \frac{p_{11}-p_{22}-2 p_{21}+\sqrt{\left(p_{11}-p_{22}\right)^{2}+4 p_{21} p_{12}}}{2\left(p_{11}+p_{12}-p_{21}-p_{22}\right)}
\end{aligned}
$$

where $\widetilde{\lambda}_{2}$ is the stable root. The following propositions assess the convergence of the difference equation (3) under all the possible parameterizations of the (primitive) transition matrix $P$. An analogous pair of roots, $\widetilde{\lambda}_{3}$ and $\widetilde{\lambda}_{4}$, with $\widetilde{\lambda}_{4}$ being the stable root, can be derived for Block 2 . Similarly, all results that follow will also apply to Block 2 .

Proposition 3 If (i) $p_{11}+p_{12}-p_{21}-p_{22} \neq 0$, (ii) $p_{11} p_{22} \neq p_{21} p_{12}$, (iii) $p_{11} \neq p_{22}$ or both $p_{12} \neq 0$ and $p_{21} \neq 0$, and the initial probability is such that $\operatorname{prob}\left(\xi_{t}=1 \mid \tau_{t}^{1}=1\right) \neq \widetilde{\lambda}_{1}$, then $\operatorname{prob}\left(\xi_{t}=1 \mid \tau_{t}^{1}\right) \longrightarrow \widetilde{\lambda}_{2} \in$ $[0,1]$. If conditions (i), (ii), and (iii) hold and the initial probability is such that prob $\left(\xi_{t}=1 \mid \tau_{t}^{1}=1\right)=\widetilde{\lambda}_{1}$, then $\operatorname{prob}\left(\xi_{t}=1 \mid \tau_{t}^{1}\right)=\widetilde{\lambda}_{1}$ for any $\tau_{t}^{1}$.

Proof. The difference equation (3) can be expressed as

$$
\operatorname{prob}\left(\xi_{t}=1 \mid \tau_{t}^{1}\right)=\frac{a \cdot \operatorname{prob}\left(\xi_{t-1}=1 \mid \tau_{t-1}^{1}\right)+b}{c \cdot \operatorname{prob}\left(\xi_{t-1}=1 \mid \tau_{t-1}^{1}\right)+d}
$$

where

$$
\begin{aligned}
a & \equiv p_{11}-p_{21}, b \equiv p_{21} \\
c & \equiv p_{11}+p_{12}-p_{21}-p_{22}, d \equiv p_{21}+p_{22}
\end{aligned}
$$

Condition (i) ensures that the difference equation of interest is rational because it implies $c>0$. We will deal with the case of $c=0$ later on. We then proceed as follows. Denote $\operatorname{prob}\left(\xi_{t}=1 \mid \tau_{t}^{1}\right)+\frac{d}{c}$ as $x_{t}$ and re-write the difference equation above as

$$
x_{t}=\alpha-\frac{\beta}{x_{t-1}}
$$

where

$$
\begin{aligned}
\alpha & \equiv \frac{p_{11}+p_{22}}{p_{11}+p_{12}-p_{21}-p_{22}} \\
\beta & \equiv \frac{p_{11} p_{22}-p_{21} p_{12}}{\left(p_{11}+p_{12}-p_{21}-p_{22}\right)^{2}}
\end{aligned}
$$

Condition (ii) ensures that $\beta \neq 0$. The case of $\beta=0$ will be studied later. The above equation can be reduced to a homogeneous linear difference equation by defining $x_{t}=\varphi_{t} / \varphi_{t-1}$ where:

$$
\varphi_{t}-\alpha \varphi_{t-1}+\beta \varphi_{t-2}=0
$$


If $\lambda_{1}$ and $\lambda_{2}$ are the solutions of the characteristic equation, namely $\frac{1}{2} \alpha \pm \frac{1}{2} \sqrt{\alpha^{2}-4 \beta}$, then the general solution of $(29)$ is

$$
\begin{aligned}
& \varphi_{t}=C_{1} \lambda_{1}^{t}+C_{2} \lambda_{2}^{t}, \quad \text { if } \lambda_{1} \neq \lambda_{2} \\
& \varphi_{t}=\left(C_{1}+C_{2} t\right) \lambda_{1}^{t}, \quad \text { if } \lambda_{1}=\lambda_{2}
\end{aligned}
$$

The general solution of $(28)$ is then:

$$
x_{t}=\frac{C_{1} \lambda_{1}^{t}+C_{2} \lambda_{2}^{t}}{C_{1} \lambda_{1}^{t-1}+C_{2} \lambda_{2}^{t-1}}
$$

when $C_{2}=0, x_{t}=\lambda_{1}$ for all $t$. When $C_{1}=0, x_{t}=\lambda_{2}$ for all $t$. When neither $C_{1}$ nor $C_{2}$ is zero, then

$$
x_{t}=\lambda_{2} \frac{\left(\frac{\lambda_{1}}{\lambda_{2}}\right)^{t+1}+C}{\left(\frac{\lambda_{1}}{\lambda_{2}}\right)^{t}+C}, C \neq 0
$$

Note that $\alpha^{2} \geq 4 \beta$ is required for the characteristic roots $\lambda_{1}$ and $\lambda_{2}$ to be real. This condition is

$$
\left[\frac{p_{11}+p_{22}}{p_{11}+p_{12}-p_{21}-p_{22}}\right]^{2} \geq 4 \frac{p_{11} p_{22}-p_{21} p_{12}}{\left(p_{11}+p_{12}-p_{21}-p_{22}\right)^{2}}
$$

and after simplifying

$$
p_{11}^{2}+p_{22}^{2}+2 p_{11} p_{22} \geq 4 p_{11} p_{22}-4 p_{21} p_{12}
$$

Some straightforward manipulation leads us to

$$
\left(p_{11}-p_{22}\right)^{2} \geq-4 p_{21} p_{12}
$$

From condition (iii), the inequality above is strict and the characteristic roots are unequal. The case in which the characteristic roots are identical is tackled by the next proposition. Let $\left|\lambda_{2}\right|>\left|\lambda_{1}\right|$ then $\left|\lambda_{1} / \lambda_{2}\right|^{t} \rightarrow 0$ and $(33)$ implies that $x_{t} \rightarrow \lambda_{2}$ as long as $x_{1} \neq \lambda_{1}$. The root with highest absolute value can be seen to be always $\frac{p_{11}+p_{22}+\sqrt{\left(p_{11}-p_{22}\right)^{2}+4 p_{21} p_{12}}}{2\left(p_{11}+p_{12}-p_{21}-p_{22}\right)}$. Recall that $x_{t} \equiv \operatorname{prob}\left(\xi_{t}=1 \mid \tau_{t}^{1}\right)+\frac{d}{c}$. After some straightforward algebraic manipulations we obtain:

$$
\operatorname{prob}\left(\xi_{t}=1 \mid \tau_{t}^{1}\right) \rightarrow \widetilde{\lambda}_{2}=\frac{p_{11}-p_{22}-2 p_{21}+\sqrt{\left(p_{11}-p_{22}\right)^{2}+4 p_{21} p_{12}}}{2\left(p_{11}+p_{12}-p_{21}-p_{22}\right)}
$$

where $\widetilde{\lambda}_{2}$ is the stable root for the variable of interest $\operatorname{prob}\left(\xi_{t}=1 \mid \tau_{t}^{1}\right)$. The unstable root for $\operatorname{prob}\left(\xi_{t}=1 \mid \tau_{t}^{1}\right)$ can be easily seen to be:

$$
\widetilde{\lambda}_{1}=\frac{p_{11}-p_{22}-2 p_{21}-\sqrt{\left(p_{11}-p_{22}\right)^{2}+4 p_{21} p_{12}}}{2\left(p_{11}+p_{12}-p_{21}-p_{22}\right)}
$$


We only need to show that $\widetilde{\lambda}_{2} \in[0,1]$. We want to show that

$$
\frac{p_{11}-p_{22}-2 p_{21}+\sqrt{\left(p_{11}-p_{22}\right)^{2}+4 p_{21} p_{12}}}{2\left(p_{11}+p_{12}-p_{21}-p_{22}\right)} \geq 0
$$

If $p_{11}+p_{12}-p_{21}-p_{22}>0$ and $p_{11}-p_{22}-2 p_{21} \geq 0$, then the statement is clearly true. When $p_{11}+p_{12}-p_{21}-p_{22}>0$ and $p_{11}-p_{22}-2 p_{21}<0$, then

$$
\sqrt{\left(p_{11}-p_{22}\right)^{2}+4 p_{21} p_{12}} \geq-\left(p_{11}-p_{22}-2 p_{21}\right)
$$

Since the right-hand side is positive we can square both sides of this equation:

$$
\begin{aligned}
\left(p_{11}-p_{22}\right)^{2}+4 p_{21} p_{12} & \geq\left(p_{11}-p_{22}-2 p_{21}\right)^{2} \\
4 p_{21} p_{12} & \geq 4 p_{21}^{2}-4\left(p_{11}-p_{22}\right) p_{21}
\end{aligned}
$$

If $p_{21}=0$, the statement is true. If $p_{21}>0$

$$
p_{12}-p_{21}+\left(p_{11}-p_{22}\right) \geq 0
$$

which is true. If $p_{11}+p_{12}-p_{21}-p_{22}<0$, then $p_{11}-p_{22}-2 p_{21}<0$. We need to show that

$$
p_{11}-p_{22}-2 p_{21} \leq-\sqrt{\left(p_{11}-p_{22}\right)^{2}+4 p_{21} p_{12}}
$$

Since both sides of the inequality are negative, then

$$
\left(p_{11}-p_{22}-2 p_{21}\right)^{2} \geq\left(p_{11}-p_{22}\right)^{2}+4 p_{21} p_{12}
$$

and after manipulating:

$$
-4\left(p_{11}-p_{22}\right) p_{21}+4 p_{21}^{2} \geq 4 p_{21} p_{12}
$$

If $p_{21}=0$, the inequality is obviously verified. If $p_{21}>0$, then

$$
0 \geq\left(p_{11}-p_{22}\right)+p_{12}-p_{21}
$$

which is true. We want to show that

$$
\frac{p_{11}-p_{22}-2 p_{21}+\sqrt{\left(p_{11}-p_{22}\right)^{2}+4 p_{21} p_{12}}}{2\left(p_{11}+p_{12}-p_{21}-p_{22}\right)} \leq 1
$$

If $p_{11}+p_{12}-p_{21}-p_{22}>0$, then after some manipulations

$$
\sqrt{\left(p_{11}-p_{22}\right)^{2}+4 p_{21} p_{12}} \leq p_{11}+2 p_{12}-p_{22}
$$


Note that $p_{11}+2 p_{12}-p_{22}>p_{11}+p_{12}-p_{21}-p_{22}>0$. Hence, taking the square on both sides of the inequality yields:

$$
\left(p_{11}-p_{22}\right)^{2}+4 p_{21} p_{12} \leq\left(p_{11}+2 p_{12}-p_{22}\right)^{2}
$$

and finally

$$
4 p_{21} p_{12} \leq 4 p_{12}^{2}+4\left(p_{11}-p_{22}\right) p_{12}
$$

If $p_{12}=0$, this is true. If $p_{12}>0$, then

$$
p_{21} \leq p_{12}+\left(p_{11}-p_{22}\right)
$$

which is true. If $p_{11}+p_{12}-p_{21}-p_{22}<0$, then after some manipulations

$$
\sqrt{\left(p_{11}-p_{22}\right)^{2}+4 p_{21} p_{12}} \geq p_{11}+2 p_{12}-p_{22}
$$

If $p_{11}+2 p_{12}-p_{22}<0$, this inequality is obviously true. If $p_{11}+2 p_{12}-p_{22} \geq 0$, then

$$
\left(p_{11}-p_{22}\right)^{2}+4 p_{21} p_{12} \geq\left(p_{11}+2 p_{12}-p_{22}\right)^{2}
$$

and then

$$
4 p_{21} p_{12} \geq 4 p_{12}^{2}+4\left(p_{11}-p_{22}\right) p_{12}
$$

If $p_{12}=0$, this is true. If $p_{12}>0$, then

$$
p_{21} \geq p_{12}+p_{11}-p_{22}
$$

which is true.

The next proposition relaxes condition (iii) of the above proposition.

Proposition 4 If (i) $p_{11}+p_{12}-p_{21}-p_{22} \neq 0$, (ii) $p_{11} p_{22} \neq p_{21} p_{12}$, (iii) $p_{11}=p_{22}$ and either $p_{12}=0$ or $p_{21}=0$, then $\operatorname{prob}\left(\xi_{t}=1 \mid \tau_{t}^{1}\right) \rightarrow \widetilde{\lambda}_{1}=\widetilde{\lambda}_{2}$ and the roots are either equal to zero (if $p_{21}=0$ ) or one (if $\left.p_{12}=0\right)$.

Proof. We want to show that if (i) $p_{11}+p_{12}-p_{21}-p_{22} \neq 0$, (ii) $p_{11} p_{22} \neq p_{21} p_{12}$, (iii) $p_{11}=p_{22}$ and either $p_{12}=0$ or $p_{21}=0$, then $\operatorname{prob}\left(\xi_{t}=1 \mid \tau_{t}^{1}\right) \rightarrow \widetilde{\lambda}_{1}=\widetilde{\lambda}_{2}$ and the roots are either equal to zero (if $p_{21}=0$ ) or one (if $p_{12}=0$ ). This result follows from observing that condition (iii) implies that condition 34 delivers coincident characteristic roots $\widetilde{\lambda}_{1}$ and $\widetilde{\lambda}_{2}$; that is,

$$
\widetilde{\lambda}_{1}=\widetilde{\lambda}_{2}=\frac{p_{21}}{p_{21}-p_{12}}
$$

If $p_{12}=0$, then $\operatorname{prob}\left(\xi_{t}=1 \mid \tau_{t}^{1}\right) \rightarrow \widetilde{\lambda}_{1}=\widetilde{\lambda}_{2}=1$. If $p_{21}=0$, then $\operatorname{prob}\left(\xi_{t}=1 \mid \tau_{t}^{1}\right) \rightarrow \widetilde{\lambda}_{1}=\widetilde{\lambda}_{2}=0$.

If the two regimes have the same persistence $\left(p_{11}=p_{22}\right)$ and the system has remained in Block 1 for sufficiently long, then agents will eventually believe they are in the regime that is an absorbing state (conditional on staying in the block). The next proposition relaxes condition ( $i i$ ) of the previous 
propositions.

Proposition 5 If (i) $p_{11}+p_{12}-p_{21}-p_{22} \neq 0$, (ii) $p_{11} p_{22}=p_{21} p_{12}$, then prob $\left(\xi_{t}=1 \mid \tau_{t}^{1}\right)=\frac{p_{11}-p_{21}}{p_{11}+p_{12}-p_{21}-p_{22}}$.

Proof. We want to show that if (i) $p_{11}+p_{12}-p_{21}-p_{22} \neq 0$, (ii) $p_{11} p_{22}=p_{21} p_{12}$, then $\operatorname{prob}\left(\xi_{t}=1 \mid \tau_{t}^{1}\right)=$ $\frac{p_{11}-p_{21}}{p_{11}+p_{12}-p_{21}-p_{22}}$. Condition (ii) implies $\beta=0$ in equation 28 and hence (using the notation introduced above)

$$
x_{t}=\alpha \equiv \frac{p_{11}+p_{22}}{p_{11}+p_{12}-p_{21}-p_{22}}
$$

Recall that $x_{t}=\operatorname{prob}\left\{\xi_{t}=1 \mid \tau_{t}^{1}\right\}+d / c$, then it follows that

$$
\operatorname{prob}\left\{\xi_{t}=1 \mid \tau_{t}^{1}\right\}=\frac{p_{11}-p_{21}}{p_{11}+p_{12}-p_{21}-p_{22}} .
$$

Note that if conditions $(i)$ and $(i i)$ are satisfied, $\operatorname{prob}\left(\xi_{t}=1 \mid \tau_{t}^{1}\right)$ suddenly converges by jumping to $\frac{p_{11}-p_{21}}{p_{11}+p_{12}-p_{21}-p_{22}}$ as the system enters Block 1 . The recursion 3 can be shown to become a linear difference equation. The solution of this equation is characterized in the following two propositions.

Proposition 6 If (i) $p_{11}+p_{12}-p_{21}-p_{22}=0$ and (ii) $p_{11} \neq p_{21}$, then $\operatorname{prob}\left(\xi_{t}=1 \mid \tau_{t}^{1}\right) \rightarrow \frac{p_{21}}{p_{22}-p_{11}+2 p_{21}}$, with $\frac{p_{21}}{p_{22}-p_{11}+2 p_{21}} \in[0,1]$.

Proof. We want to show that if (i) $p_{11}+p_{12}-p_{21}-p_{22}=0$ and (ii) $p_{11} \neq p_{21}$, then $\operatorname{prob}\left(\xi_{t}=1 \mid \tau_{t}^{1}\right) \rightarrow$ $\frac{p_{21}}{p_{22}-p_{11}+2 p_{21}}$, with $\frac{p_{21}}{p_{22}-p_{11}+2 p_{21}} \in[0,1]$. If $p_{11}+p_{12}-p_{21}-p_{22}=0$, then $c=0$ in the difference equation (27), which hence boils down to the first-order linear difference equation below:

$$
\operatorname{prob}\left(\xi_{t}=1 \mid \tau_{t}^{1}\right)=\frac{a}{d} \cdot \operatorname{prob}\left(\xi_{t-1}=1 \mid \tau_{t-1}^{1}\right)+\frac{b}{d}
$$

where $a=p_{11}-p_{21}, \quad b=p_{21}, d=p_{21}+p_{22}$. Stability is ensured by $\left|\frac{a}{d}\right|=\left|\frac{p_{11}-p_{21}}{p_{21}+p_{22}}\right|<1$. First note that the benchmark assumption A1 combined with condition (i) implies that $d \neq 0$ and hence the ratio $\left|\frac{a}{d}\right|$ is well-defined. Condition (ii) rules out the possibility that the ratio $\left|\frac{a}{d}\right|$ is zero. We will tackle this case in the next proposition. The condition $p_{11}+p_{12}-p_{21}-p_{22}=0$ allows us to re-write the stability condition $\left|\frac{a}{d}\right|=\left|\frac{p_{11}-p_{21}}{p_{21}+p_{22}}\right|$ as $\left|\frac{p_{11}-p_{21}}{p_{11}+p_{12}}\right|$. Hence, showing that $p_{12}+p_{21}>0$ implies stability. Recall that the benchmark assumption $\mathrm{A} 2$ requires that either $p_{11} \neq p_{22}$ or $p_{12} \neq p_{21}$. If the latter condition is satisfied, then $p_{12}+p_{21}>0$ trivially follows. If the latter condition is not satisfied, then it must be that $p_{11} \neq p_{22}$, which, combined with condition (i), implies that $p_{12}+p_{21}>0$. It is easy to see that the difference equation 35 implies that $\operatorname{prob}\left(\xi_{t}=1 \mid \tau_{t}^{1}\right) \rightarrow \frac{b}{d}\left(1-\frac{a}{d}\right)^{-1}$, that is,

$$
\operatorname{prob}\left(\xi_{t}=1 \mid \tau_{t}^{1}\right) \rightarrow \frac{p_{21}}{p_{21}+p_{22}}\left(1-\frac{p_{11}-p_{21}}{p_{21}+p_{22}}\right)^{-1}
$$

After easy algebraic manipulations

$$
\operatorname{prob}\left(\xi_{t}=1 \mid \tau_{t}^{1}\right) \rightarrow \frac{p_{21}}{p_{22}-p_{11}+2 p_{21}}
$$


Note that

$$
0 \leq \frac{p_{21}}{p_{22}-p_{11}+2 p_{21}} \leq 1
$$

To see that, recall that in this case, $p_{11}+p_{12}-p_{21}-p_{22}=0$, implying that $p_{22}-p_{11}=p_{12}-p_{21}$. Substituting this result into the inequalities above yields

$$
0 \leq \frac{p_{21}}{p_{12}+p_{21}} \leq 1
$$

which is clearly verified.

Proposition 7 If (i) $p_{11}+p_{12}-p_{21}-p_{22}=0$, (ii) $p_{11}=p_{21}$, then prob $\left(\xi_{t}=1 \mid \tau_{t}^{1}\right)=\frac{p_{21}}{p_{22}+p_{21}}$.

Proof. We want to show that if (i) $p_{11}+p_{12}-p_{21}-p_{22}=0$, (ii) $p_{11}=p_{21}$, then $\operatorname{prob}\left(\xi_{t}=1 \mid \tau_{t}^{1}\right)=\frac{p_{21}}{p_{22}+p_{21}}$. Condition (i) implies that $c=0$ in the difference equation (27), which hence boils down to the first-order linear difference equation below:

$$
\operatorname{prob}\left(\xi_{t}=1 \mid \tau_{t}^{1}\right)=\frac{a}{d} \cdot \operatorname{prob}\left(\xi_{t-1}=1 \mid \tau_{t-1}^{1}\right)+\frac{b}{d}
$$

where $a=p_{11}-p_{21}, b=p_{21}, d=p_{21}+p_{22}$. Condition (ii) implies that $a=0$ and hence prob $\left(\xi_{t}=1 \mid \tau_{t}^{1}\right)=$ $b / d=p_{21} /\left(p_{21}+p_{22}\right)$.

It should be noted that when $p_{11}=p_{21}$, beliefs $\operatorname{prob}\left(\xi_{t}=1 \mid \tau_{t}^{1}\right)$ suddenly jump to $\frac{p_{21}}{p_{22}+p_{21}}$ for any $\tau_{t}^{1} \geq 1$ (as the system enters Block 1 ).

To sum up, given the benchmark assumptions $A 1-A 3$, we have shown that equation (3) always converges. Note that Proposition 2 implies that beliefs do not converge to $\widetilde{\lambda}_{2}$, if the starting beliefs $\operatorname{prob}\left(\xi_{t}=1 \mid \tau_{t}^{1}=1\right)=\widetilde{\lambda}_{1}$. The next two propositions show that either $\widetilde{\lambda}_{1} \leq 0$ or $\widetilde{\lambda}_{1} \geq 1$, implying that the only admissible values for probabilities are either zero or one. Therefore, there are only a few limiting cases in which equation (3) does not converge to $\tilde{\lambda}_{2}$. It can be shown that it is sufficient to set the probability ratios $0<p_{i 3} /\left(p_{i 3}+p_{i 4}\right)<1$ for any $i \in\{1,2\}$ to rule out these cases that are not very relevant in practice.

Recall that

$$
\widetilde{\lambda}_{1} \equiv \frac{p_{11}-p_{22}-2 p_{21}-\sqrt{\left(p_{11}-p_{22}\right)^{2}+4 p_{21} p_{12}}}{2\left(p_{11}+p_{12}-p_{21}-p_{22}\right)}
$$

We want to show that $0 \leq \widetilde{\lambda}_{1} \leq 1$. This claim is implied by the following two propositions.

Proposition 8 If $p_{11}+p_{12}-p_{21}-p_{22}>0$, then $\widetilde{\lambda}_{1} \leq 0$.

Proof. We want to show that

$$
\frac{p_{11}-p_{22}-2 p_{21}-\sqrt{\left(p_{11}-p_{22}\right)^{2}+4 p_{21} p_{12}}}{2\left(p_{11}+p_{12}-p_{21}-p_{22}\right)} \leq 0
$$

If $p_{11}+p_{12}-p_{21}-p_{22}>0$, then the above implies

$$
p_{11}-p_{22}-2 p_{21} \leq \sqrt{\left(p_{11}-p_{22}\right)^{2}+4 p_{21} p_{12}}
$$


Note that the benchmark assumption A3 excludes that $p_{11}-p_{22}-2 p_{21}=0$. Hence there are two possible cases left: (a) if $p_{11}-p_{22}-2 p_{21}<0$, then the above is true; $(b)$ if $p_{11}-p_{22}-2 p_{21}>0$, then we can take the square on both sides of the above equation to get

$$
\left(p_{11}-p_{22}-2 p_{21}\right)^{2} \leq\left(p_{11}-p_{22}\right)^{2}+4 p_{21} p_{12}
$$

Straightforward manipulations lead to

$$
p_{21}^{2}-p_{11} p_{21}+p_{22} p_{21} \leq p_{21} p_{12}
$$

If $p_{21}=0$, then the above is true. Otherwise, we can divide both sides of the above inequality by $p_{21}$ to get

$$
p_{11}+p_{12}-p_{21}-p_{22} \geq 0
$$

that is obviously true because $p_{11}+p_{12}-p_{21}-p_{22}>0$.

Proposition 9 If $p_{11}+p_{12}-p_{21}-p_{22}<0$, then $\widetilde{\lambda}_{1} \geq 1$.

Proof. We want to show that

$$
\frac{p_{11}-p_{22}-2 p_{21}-\sqrt{\left(p_{11}-p_{22}\right)^{2}+4 p_{21} p_{12}}}{2\left(p_{11}+p_{12}-p_{21}-p_{22}\right)} \geq 1
$$

Since $p_{11}+p_{12}-p_{21}-p_{22}<0$, the above implies

$$
p_{11}-p_{22}-2 p_{21}-\sqrt{\left(p_{11}-p_{22}\right)^{2}+4 p_{21} p_{12}} \leq 2\left(p_{11}+p_{12}-p_{21}-p_{22}\right)
$$

and after simplifying

$$
-\sqrt{\left(p_{11}-p_{22}\right)^{2}+4 p_{21} p_{12}} \leq p_{11}-p_{22}+2 p_{12}
$$

Note that the benchmark assumption A3 excludes that $p_{11}-p_{22}+2 p_{21}=0$. If $p_{11}-p_{22}+2 p_{12}>0$, the above is obviously true. If $p_{11}-p_{22}+2 p_{12}<0$, then taking the square on both sides

$$
\left(p_{11}-p_{22}\right)^{2}+4 p_{21} p_{12} \geq\left(p_{11}-p_{22}+2 p_{12}\right)^{2}
$$

After some manipulations:

$$
p_{12}+p_{11}-p_{12}-p_{22} \leq 0
$$

that is obviously true because $p_{11}+p_{12}-p_{21}-p_{22}<0$.

\section{Algorithm for the Case with Signals}

Algorithm Set $i=1$ and initialize the matrix $\widehat{\mathcal{P}}=\mathbf{0}_{g \times g}$

Step 1 Find $j_{1} \leq g_{1}$ and $j_{2} \leq g_{1}$ so as to $\min \left|\operatorname{prob}\left\{\xi_{t}=1 \mid \mathcal{I}_{t}, \varpi^{t-1}, \varpi_{t}=q\right\}-\mathcal{G}_{j_{q}}\right|$ with $q \in\{1,2\}$ 
where

$$
\operatorname{prob}\left(\xi_{t}=1 \mid \mathcal{I}_{t}, \varpi^{t-1}, \varpi_{t}=q\right)=\frac{\operatorname{prob}\left(\varpi_{t}=q \mid \xi_{t}=1\right) \operatorname{prob}\left(\xi_{t}=1 \mid \mathcal{I}_{t}, \varpi^{t-1}\right)}{\sum_{j=1}^{2} \operatorname{prob}\left(\varpi_{t}=q \mid \xi_{t}=j\right) \operatorname{prob}\left(\xi_{t}=j \mid \mathcal{I}_{t}, \varpi^{t-1}\right)}, q \in\{1,2\}
$$

and agents' beliefs about being in Regime 1 before observing the signal read:

$$
\operatorname{prob}\left(\xi_{t}=1 \mid \mathcal{I}_{t}, \varpi^{t-1}\right)=\frac{\operatorname{prob}\left(\xi_{t-1}=1 \mid \mathcal{I}_{t-1}, \varpi^{t-1}\right)\left(p_{11}-p_{21}\right)+p_{21}}{\operatorname{prob}\left(\xi_{t-1}=1 \mid \mathcal{I}_{t-1}, \varpi^{t-1}\right)\left(p_{11}+p_{12}-p_{21}-p_{22}\right)+p_{21}+p_{22}}
$$

using the approximation $\operatorname{prob}\left\{\xi_{t-1}=1 \mid \mathcal{I}_{t-1}, \varpi^{t-1}\right\}=\mathcal{G}_{i}$. To ensure convergence of beliefs, we correct $j_{1}$ and $j_{2}$ as follows. If $j_{q}=i$ and $\mathcal{G}_{i} \neq \widetilde{\lambda}_{2}(q \in\{1,2\})$, then set $j_{q}=j_{q}+1$ if $\mathcal{G}_{i}<\widetilde{\lambda}_{2}$ and $j_{q}=\max \left(1, j_{q}-1\right)$ if $\mathcal{G}_{i}>\tilde{\lambda}_{2}$.

Step 2 Setting $\operatorname{prob}\left(\xi_{t-1}=1 \mid \mathcal{I}_{t-1}, \varpi^{t-1}\right)=\mathcal{G}_{i}$, the (ex-ante) transition probability can be computed as:

$$
\widehat{\mathcal{P}}\left(i, j_{q}\right)=\sum_{v=1}^{2} \operatorname{prob}\left\{\xi_{t}=v \mid \mathcal{I}_{t-1}, \varpi^{t-1}\right\} \operatorname{prob}\left\{\varpi_{t}=q \mid \xi_{t}=v\right\}, \quad q \in\{1,2\}
$$

where

$$
\operatorname{prob}\left\{\xi_{t}=v \mid \mathcal{I}_{t-1}, \varpi^{t-1}\right\}=\sum_{u=1}^{2} \operatorname{prob}\left\{\xi_{t-1}=u \mid \mathcal{I}_{t-1}, \varpi^{t-1}\right\} p_{u v}
$$

Step 3 Find $j_{1}>g_{1}$ and $j_{2}>g_{1}$ so as to $\min \left|\operatorname{prob}\left\{\xi_{t}=3 \mid \mathcal{I}_{t}, \varpi^{t-1}, \varpi_{t}=q\right\}-\mathcal{G}_{j_{q}}\right|$ with $q \in\{1,2\}$, where

$$
\operatorname{prob}\left(\xi_{t}=3 \mid \mathcal{I}_{t}, \varpi^{t-1}, \varpi_{t}=q\right)=\frac{\operatorname{prob}\left(\varpi_{t}=q \mid \xi_{t}=3\right) \operatorname{prob}\left(\xi_{t}=3 \mid \mathcal{I}_{t}, \varpi^{t-1}\right)}{\sum_{j=3}^{4} \operatorname{prob}\left(\varpi_{t}=q \mid \xi_{t}=j\right) \operatorname{prob}\left(\xi_{t}=j \mid \mathcal{I}_{t}, \varpi^{t-1}\right)}, q \in\{1,2\}
$$

and the beliefs about being in Regime 3 upon the shift to Block 2 (before having observed the signal $\left.\varpi_{t}\right)$ are given by:

$$
\begin{gathered}
\operatorname{prob}\left\{\xi_{t}=3 \mid \mathcal{I}_{t}, \varpi^{t-1}\right\}=\frac{\sum_{j \in b_{1}} \operatorname{prob}\left\{\xi_{t-1}=j \mid \mathcal{I}_{t-1}, \varpi^{t-1}\right\} p_{j 3}}{\sum_{i \in b_{2}} \sum_{j \in b_{1}} \operatorname{prob}\left\{\xi_{t-1}=j \mid \mathcal{I}_{t-1}, \varpi^{t-1}\right\} p_{j i}} \\
=\frac{\operatorname{prob}\left\{\xi_{t-1}=1 \mid \mathcal{I}_{t-1}, \varpi^{t-1}\right\} p_{13}+\left(1-\operatorname{prob}\left\{\xi_{t-1}=1 \mid \mathcal{I}_{t-1}, \varpi^{t-1}\right\}\right) p_{23}}{\operatorname{prob}\left\{\xi_{t-1}=1 \mid \mathcal{I}_{t-1}, \varpi^{t-1}\right\}\left(p_{13}+p_{14}\right)+\left(1-\operatorname{prob}\left\{\xi_{t-1}=1 \mid \mathcal{I}_{t-1}, \varpi^{t-1}\right\}\right)\left(p_{23}+p_{24}\right)}
\end{gathered}
$$

using the approximation that $\operatorname{prob}\left(\xi_{t-1}=1 \mid \mathcal{I}_{t-1}, \varpi^{t-1}\right)=\mathcal{G}_{i}$. Setting $\operatorname{prob}\left(\xi_{t-1}=1 \mid \mathcal{I}_{t-1}, \varpi^{t-1}\right)=$ $\mathcal{G}_{i}$, the (ex-ante) transition probabilities as

$$
\widehat{\mathcal{P}}\left(i, j_{q}\right)=\widehat{\mathcal{P}}\left(i, j_{q}\right)+\sum_{v=3}^{4}\left(\sum_{u=1}^{2} \operatorname{prob}\left\{\xi_{t-1}=u \mid \mathcal{I}_{t-1}, \varpi^{t-1}\right\} p_{u v}\right) \operatorname{prob}\left\{\varpi_{t}=q \mid \xi_{t}=v\right\}, \quad q \in\{1,2\}
$$

Step 4 If $i=g_{1}$ then set $i=i+1$ and go to step 6 ; otherwise, set $i=i+1$ and go to step 1 .

Step 5 Find $j_{1}>g_{1}$ and $j_{2}>g_{1}$ so as to min $\left|\operatorname{prob}\left\{\xi_{t}=3 \mid \mathcal{I}_{t}, \varpi^{t-1}, \varpi_{t}=q\right\}-\mathcal{G}_{j_{q}}\right|$ with $q \in\{1,2\}$ 
where

$$
\operatorname{prob}\left(\xi_{t}=3 \mid \mathcal{I}_{t}, \varpi^{t-1}, \varpi_{t}=q\right)=\frac{\operatorname{prob}\left(\varpi_{t}=q \mid \xi_{t}=3\right) \operatorname{prob}\left(\xi_{t}=3 \mid \mathcal{I}_{t}, \varpi^{t-1}\right)}{\sum_{j=3}^{4} \operatorname{prob}\left(\varpi_{t}=q \mid \xi_{t}=j\right) \operatorname{prob}\left(\xi_{t}=j \mid \mathcal{I}_{t}, \varpi^{t-1}\right)}, q \in\{1,2\}
$$

and agents' beliefs about being in Regime 3 before observing the signal read:

$$
\operatorname{prob}\left(\xi_{t}=3 \mid \mathcal{I}_{t}, \varpi^{t-1}\right)=\frac{\operatorname{prob}\left(\xi_{t-1}=3 \mid \mathcal{I}_{t-1}, \varpi^{t-1}\right)\left(p_{33}-p_{43}\right)+p_{43}}{\operatorname{prob}\left(\xi_{t-1}=3 \mid \mathcal{I}_{t-1}, \varpi^{t-1}\right)\left(p_{33}+p_{34}-p_{43}-p_{44}\right)+p_{43}+p_{44}}
$$

using the approximation $\operatorname{prob}\left\{\xi_{t-1}=3 \mid \mathcal{I}_{t-1}, \varpi^{t-1}\right\}=\mathcal{G}_{i}$. To ensure convergence of beliefs, we correct $j_{1}$ and $j_{2}$ as follows. If $j_{q}=i$ and $\mathcal{G}_{i} \neq \widetilde{\lambda}_{4}(q \in\{1,2\})$, then set $j_{q}=\min \left(j_{q}+1, g\right)$ if $\mathcal{G}_{i}<\widetilde{\lambda}_{4}$ and $j_{q}=j_{q}-1$ if $\mathcal{G}_{i}>\widetilde{\lambda}_{4}$.

Step 6 Setting $\operatorname{prob}\left(\xi_{t-1}=3 \mid \mathcal{I}_{t-1}, \varpi^{t-1}\right)=\mathcal{G}_{i}$, the (ex-ante) transition probability can be computed as:

$$
\widehat{\mathcal{P}}\left(i, j_{q}\right)=\widehat{\mathcal{P}}\left(i, j_{q}\right)+\sum_{v=3}^{4}\left(\sum_{u=3}^{4} \operatorname{prob}\left\{\xi_{t-1}=u \mid \mathcal{I}_{t-1}, \varpi^{t-1}\right\} p_{u v}\right) \operatorname{prob}\left\{\varpi_{t}=q \mid \xi_{t}=v\right\}, \quad q \in\{1,2\}
$$

Step 7 Find $j_{1} \leq g_{1}$ and $j_{2} \leq g_{1}$ so as to $\min \left|\operatorname{prob}\left\{\xi_{t}=1 \mid \mathcal{I}_{t}, \varpi^{t-1}, \varpi_{t}=q\right\}-\mathcal{G}_{j_{q}}\right|$ with $q \in\{1,2\}$, where

$$
\operatorname{prob}\left(\xi_{t}=1 \mid \mathcal{I}_{t}, \varpi^{t-1}, \varpi_{t}=q\right)=\frac{\operatorname{prob}\left(\varpi_{t}=q \mid \xi_{t}=1\right) \operatorname{prob}\left(\xi_{t}=1 \mid \mathcal{I}_{t}, \varpi^{t-1}\right)}{\sum_{j=1}^{2} \operatorname{prob}\left(\varpi_{t}=q \mid \xi_{t}=j\right) \operatorname{prob}\left(\xi_{t}=j \mid \mathcal{I}_{t}, \varpi^{t-1}\right)}, q \in\{1,2\}
$$

and the beliefs about being in Regime 1 upon the shift to Block 1 (before having observed the signal $\left.\varpi_{t}\right)$ are given by:

$$
\begin{gathered}
\quad \operatorname{prob}\left\{\xi_{t}=1 \mid \mathcal{I}_{t}, \varpi^{t-1}\right\}=\frac{\sum_{j \in b_{2}} \operatorname{prob}\left\{\xi_{t-1}=j \mid \mathcal{I}_{t-1}, \varpi^{t-1}\right\} p_{j 1}}{\sum_{i \in b_{1}} \sum_{j \in b_{2}} \operatorname{prob}\left\{\xi_{t-1}=j \mid \mathcal{I}_{t-1}, \varpi^{t-1}\right\} p_{j i}} \\
=\frac{\operatorname{prob}\left\{\xi_{t-1}=3 \mid \mathcal{I}_{t-1}, \varpi^{t-1}\right\} p_{31}+\left(1-\operatorname{prob}\left\{\xi_{t-1}=3 \mid \mathcal{I}_{t-1}, \varpi^{t-1}\right\}\right) p_{41}}{\operatorname{prob}\left\{\xi_{t-1}=3 \mid \mathcal{I}_{t-1}, \varpi^{t-1}\right\}\left(p_{31}+p_{32}\right)+\left(1-\operatorname{prob}\left\{\xi_{t-1}=3 \mid \mathcal{I}_{t-1}, \varpi^{t-1}\right\}\right)\left(p_{41}+p_{42}\right)}
\end{gathered}
$$

using the approximation that $\operatorname{prob}\left(\xi_{t-1}=3 \mid \mathcal{I}_{t-1}, \varpi^{t-1}\right)=\mathcal{G}_{g_{1}+i}$. Setting $\operatorname{prob}\left(\xi_{t-1}=3 \mid \mathcal{I}_{t-1}, \varpi^{t-1}\right)=$ $\mathcal{G}_{i}$, the (ex-ante) transition probability can be computed as:

$$
\widehat{\mathcal{P}}\left(i, j_{q}\right)=\widehat{\mathcal{P}}\left(i, j_{q}\right)+\sum_{v=1}^{2}\left(\sum_{u=3}^{4} \operatorname{prob}\left\{\xi_{t-1}=u \mid \mathcal{I}_{t-1}, \varpi^{t-1}\right\} p_{u v}\right) \operatorname{prob}\left\{\varpi_{t}=q \mid \xi_{t}=v\right\}, \quad q \in\{1,2\}
$$

Step 8 If $i=g$, then go to step 9; otherwise, set $i=i+1$ and go to step 5 .

Step 9 If no column of $\widehat{\mathcal{P}}$ has all zero elements, then stop. Otherwise, go to step 10.

Step 10 Construct the matrix $T$ as follows. Set $j=1$ and $l=1$. While $j \leq g$, if $\sum_{i=1}^{g} \widehat{\mathcal{P}}(i, j) \neq 0$ then do three things: $(1)$ set $T(j, l)=1,(2)$ set $T(j, v)=0$ for any $1 \leq v \leq g$ and $v \neq l,(3)$ set $l=l+1$ 
and (4) set $j=j+1$; otherwise (i.e., if $\sum_{i=1}^{g} \widehat{\mathcal{P}}(i, j)=0$ ), set $j=j+1$.

Step 11 Write the transition equation as $\widehat{\mathcal{P}}^{R}=T \cdot \widehat{\mathcal{P}} \cdot T^{\prime}$. If no column of $\widehat{\mathcal{P}}^{R}$ has all zero elements, set $\widehat{\mathcal{P}}=\widehat{\mathcal{P}}^{R}$ and stop. Otherwise, go to step 10 .

\section{Log-Linearization of the RBC Model}

Solving the problem of the representative household in Section 3 leads to:

$$
\begin{aligned}
c_{t}^{-1} & =\beta \widetilde{E}_{t} c_{t+1}^{-1}\left[\alpha z_{t+1} k_{t}^{\alpha-1}+1-\delta\right] \\
c_{t}+k_{t} & =z_{t} k_{t-1}^{\alpha}+(1-\delta) k_{t-1}
\end{aligned}
$$

The stochastic process of TFP (17) and equations (45)-(46) imply that consumption and capital are non-stationary. Denote the stationary variables $\widetilde{c}_{t} \equiv c_{t} / z_{t}^{(1-\alpha)^{-1}}, \widetilde{k}_{t} \equiv k_{t} / z_{t}^{(1-\alpha)^{-1}}, \mu_{t} \equiv \ln \left(z_{t} / z_{t-1}\right)$, and $M_{t} \equiv z_{t} / z_{t-1}$ as the gross growth rate of TFP. The stationary version of the model reads:

$$
\begin{aligned}
\widetilde{c}_{t}^{-1} & =\beta \widetilde{E}_{t} \widetilde{c}_{t+1}^{-1} M_{t+1}^{\frac{1}{\alpha-1}}\left[\alpha M_{t+1} \widetilde{k}_{t}^{\alpha-1}+1-\delta\right] \\
\widetilde{c}_{t}+\widetilde{k}_{t} & =M \widetilde{k}_{t-1}^{\alpha}+(1-\delta) M_{t}^{\frac{1}{\alpha-1}} \widetilde{k}_{t-1}
\end{aligned}
$$

Following Schorfheide (2005) and Liu et al. (2011), we define a steady-state equilibrium for the stationary consumption $\widetilde{c}_{t}$ and capital $\widetilde{k}_{t}$ when $\varepsilon_{t}=0$ all $t$ and the growth rate of TFP is at its ergodic value $\bar{\mu}$. The steady-state equilibrium level of consumption $c_{s s}$ and capital $k_{s s}$ is:

$$
\begin{aligned}
k_{s s} & =\left[\frac{1}{\alpha \bar{M}}\left(\frac{\bar{M}^{\frac{1}{1-\alpha}}}{\beta}-1+\delta\right)\right]^{\frac{1}{\alpha-1}} \\
c_{s s} & =\bar{M}^{\frac{\alpha}{\alpha-1}} k_{s s}^{\alpha}+\left[(1-\delta) \bar{M}^{\frac{1}{\alpha-1}}-1\right] k_{s s}
\end{aligned}
$$

where $\bar{M} \equiv \exp (\bar{\mu}), \bar{\mu} \equiv\left(\bar{p}_{1}+\bar{p}_{2}\right) \mu_{H}+\left(\bar{p}_{3}+\bar{p}_{4}\right) \mu_{L}$ is the ergodic mean of the log growth rate of the economy, and $\bar{p}_{i}$ stands for the ergodic probability of being in Regime $i$.

Taking the log-linear approximation of equations (47)-(48) around the steady-state equilibrium (49)(50) leads to

$$
\widehat{c}_{t}=\widetilde{E}_{t} \widehat{c}_{t+1}-(\alpha-1)\left(1+(\delta-1) \beta \bar{M}^{\frac{1}{\alpha-1}}\right) \widehat{k}_{t}-\left(\frac{1}{\alpha-1}+\beta \bar{M}^{\frac{1}{\alpha-1}}(\delta-1)+1\right) \widetilde{E}_{t} \widehat{\mu}_{t+1}
$$

where we use the fact that $\beta \bar{M}^{\frac{1}{\alpha-1}}\left(\alpha \bar{M} k_{s s}^{\alpha-1}+1-\delta\right)=1$ from equation 49 and $\widehat{\mu}_{t} \equiv \mu_{t}-\bar{\mu}$ is the log-deviation of the growth rate of TFP from its ergodic mean $\bar{\mu}$. $\widehat{c}_{t}$ and $\widehat{k}_{t}$ denote log-deviations of the stationary consumption and capital, respectively, from their steady-state value, and $\widehat{\mu}\left(\xi_{t}\right) \equiv \mu_{t}\left(\xi_{t}\right)-\bar{\mu}$ 
is the log-deviation of the TFP drift from its ergodic mean $\bar{\mu}$. The resource constraint is

$$
c_{s s} \widehat{c}_{t}+k_{s s} \widehat{k}_{t}=\left(\bar{M}^{\frac{\alpha}{\alpha-1}} k_{s s}^{\alpha} \frac{\alpha}{\alpha-1}+\frac{1-\delta}{\alpha-1} \bar{M}^{\frac{1}{\alpha-1}} k_{s s}\right) \widehat{\mu}_{t}+\left(\bar{M}^{\frac{\alpha}{\alpha-1}} k_{s s}^{\alpha} \alpha+(1-\delta) \bar{M}^{\frac{1}{\alpha-1}} k_{s s}\right) \widehat{k}_{t-1}
$$

and the log-deviations of the growth rate of TFP from its ergodic level follows

$$
\widehat{\mu}_{t}=\widehat{\mu}_{t}\left(\xi_{t}\right)+\sigma_{z} \varepsilon_{t}
$$

\section{References}

Barlevy, G. (1998). Learning costs and delayed adjustment. Manuscript.

Bianchi, F. (2013a). Methods for measuring expectations and uncertainty in markov-switching models. Manuscript.

Bianchi, F. (2013b). Regime switches, agents' beliefs, and post-world war ii u.s. macroeconomic dynamics. Review of Economic Studies 80(2), 463-490.

Bianchi, F., C. L. Ilut, and M. Schneider (2014, May). Uncertainty Shocks, Asset Supply and Pricing over the Business Cycle. NBER Working Papers 20081, National Bureau of Economic Research, Inc.

Bianchi, F. and L. Melosi (2014). Dormant shocks and fiscal virtue. In J. Parker and M. Woodford (Eds.), NBER Macroeconomics Annual 2013, Volume 28 of NBER Chapters, pp. 1-46. National Bureau of Economic Research, Inc.

Bianchi, F. and L. Melosi (2015). Constrained discretion and central bank transparency. Manuscript.

Blanchard, O. J. and C. M. Kahn (1980, July). The Solution of Linear Difference Models under Rational Expectations. Econometrica 48(5), 1305-11.

Cho, S. (2014). Sufficient Conditions for Determinacy on a Class of Markov-Switching Rational Expectations Models. working paper.

Christiano, L. J., M. Eichenbaum, and C. L. Evans (2005). Nominal rigidities and the dynamic effects of a shock to monetary policy. Journal of Political Economy 113(1), 1-45.

Cogley, T., C. Matthes, and A. M. Sbordone (2011). Optimal disinflation under learning. Staff Reports 524, Federal Reserve Bank of New York.

Cogley, T. and T. J. Sargent (2005, April). Drift and volatilities: Monetary policies and outcomes in the post wwii u.s. Review of Economic Dynamics 8(2), 262-302.

Cogley, T. and T. J. Sargent (2008, 02). Anticipated Utility And Rational Expectations As Approximations Of Bayesian Decision Making. International Economic Review 49(1), 185-221.

Davig, T. and T. Doh (2014, December). Monetary Policy Regime Shifts and Inflation Persistence. The Review of Economics and Statistics 96(5), 862-875. 
Davig, T. and E. M. Leeper (2007, June). Generalizing the taylor principle. American Economic Review $97(3), 607-635$.

Del Negro, M. and S. Eusepi (2011). Fitting observed inflation expectations. Journal of Economic Dynamics and Control 35(12), 2105-2131.

Edge, R. M., T. Laubach, and J. C. Williams (2007, November). Learning and shifts in long-run productivity growth. Journal of Monetary Economics 54(8), 2421-2438.

Evans, G. W. and S. Honkapohja (2001). Learning and Expectations in Macroeconomics. Princeton University Press.

Farmer, R. E., D. F. Waggoner, and T. Zha (2009, September). Understanding markov-switching rational expectations models. Journal of Economic Theory 144(5), 1849-1867.

Fernandez-Villaverde, J. and J. F. Rubio-Ramirez (2008, January). How structural are structural parameters? In NBER Macroeconomics Annual 2007, Volume 22, NBER Chapters, pp. 83-137. National Bureau of Economic Research, Inc.

Foerster, A., J. Rubio-Ramirez, D. Waggoner, and T. Zha (2013). Perturbation methods for Markovswitching DSGE model. Technical report.

Justiniano, A. and G. E. Primiceri (2008, June). The time-varying volatility of macroeconomic fluctuations. American Economic Review 98(3), 604-41.

Liu, Z., D. F. Waggoner, and T. Zha (2011, 07). Sources of macroeconomic fluctuations: A regimeswitching DSGE approach. Quantitative Economics 2(2), 251-301.

Mankiw, N. G., R. Reis, and J. Wolfers (2004, January). Disagreement about inflation expectations. In NBER Macroeconomics Annual 2003, Volume 18, NBER Chapters, pp. 209-270. National Bureau of Economic Research, Inc.

Melosi, L. (2014a). Estimating models with dispersed information. American Economic Journal: Macroeconomics $6(1), 1-31$.

Melosi, L. (2014b). Signaling effects of monetary policy. Mimeo Federal Reserve Bank of Chicago.

Nimark, K. (2008). Dynamic pricing and imperfect common knowledge. Journal of Monetary Economics 55(8), 365-382.

Primiceri, G. E. (2005). Time varying structural vector autoregressions and monetary policy. Review of Economic Studies 72(3), 821-852.

Schorfheide, F. (2005, April). Learning and monetary policy shifts. Review of Economic Dynamics 8(2), $392-419$.

Sims, C. A. (2002, October). Solving linear rational expectations models. Computational Economics 20(12), 1-20. 
Sims, C. A. and T. Zha (2006, March). Were there regime switches in u.s. monetary policy? American Economic Review 96(1), 54-81.

Smets, F. and R. Wouters (2007). Shocks and frictions in us business cycles: A bayeasian dsge approach. American Economic Review 97(3), 586-606. 